TRANSACTIONS OF THE

AMERICAN MATHEMATICAL SOCIETY

Volume 354, Number 12, Pages 4921-4951

S 0002-9947(02)03077-5

Article electronically published on July 23, 2002

\title{
A MEASURE-VALUED ANALOGUE OF WIENER MEASURE AND THE MEASURE-VALUED FEYNMAN-KAC FORMULA
}

\author{
K. S. RYU AND M. K. IM
}

Dedicated to Professor Kun Soo Chang on his sixtieth birthday

\begin{abstract}
In this article, we consider a complex-valued and a measure-valued measure on $C[0, t]$, the space of all real-valued continuous functions on $[0, t]$. Using these concepts, we establish the measure-valued Feynman-Kac formula and we prove that this formula satisfies a Volterra integral equation. The work here is patterned to some extent on earlier works by Kluvanek in 1983 and by Lapidus in 1987, but the present setting requires a number of new concepts and results.
\end{abstract}

\section{INTRODUCTION}

In 1923, Wiener showed that one can define a reasonable measure on the space $C_{0}[0, t]$ of all continuous functions on a closed interval $[0, t]$ that vanish at the origin 0 , the so-called Wiener space [20]. Since then, the theory of this measure was investigated extensively and applied to various subjects by many mathematicians and many mathematical physicists. In 1968, Cameron and Storvick presented the definitions and theories for the operator-valued function space integral on $C_{0}[0, t]$ [2]. In 1986, Johnson and Lapidus obtained a perturbation expansion for the operatorvalued function space integral under quite general conditions. Further, in 1987, Lapidus showed that for an exponential functional, the result in their paper satisfies a Volterra integral equation [9, 12, [13, 14, thereby establishing in this context the Feynman-Kac formula with a (complex-valued) measure. In 1983, Kluvanek introduced a measure $\mu_{\varphi}$ on the space $C_{0}[0, t]$ of all continuous functions on a closed interval by a method similar to the Wiener case and introduced an operator-valued measure $M_{t}$ on $C_{0}[0, t]$ associated with a measure $\mu_{\varphi}$ [10]. Moreover, he found an operator-valued Feynman-Kac formula with respect to an operator-valued measure $M_{t}$ and proved that his results satisfy a Volterra integral equation.

In this article, we introduce a complex-valued analogue of Wiener measure $\omega_{\varphi}$ on $C[0, t]$, associated with a complex-valued measure $\varphi$ on $\mathbb{R}$. Indeed, if $\varphi$ is the Dirac measure $\delta_{0}$ at the origin in $\mathbb{R}, \omega_{\varphi}$ is the concrete Wiener measure. Using these concepts and the concepts of the conditional Wiener integral [21, we derive the measure-valued measure $V_{\varphi}$ on $C[0, t]$ and establish the measure-valued FeynmanKac formula. Moreover, we prove that our results - the measure-valued FeynmanKac formula - satisfy a Volterra integral equation.

Received by the editors December 18, 2001 and, in revised form, April 1, 2002.

2000 Mathematics Subject Classification. Primary 28C35, 28C20, 45D05, 47A56.

Key words and phrases. Analogue of Wiener measure, Bartle integral, measure-valued Feynman-Kac formula, Volterra integral equation. 
This article consists of six sections. In section 2, we introduce some notation, some definitions and some basic facts which are needed to understand the contents of the subsequent sections. In section 3, we define a complex-valued analogue of Wiener measure $\omega_{\varphi}$ on $C[0, t]$, associated with a complex Borel measure $\varphi$ on $\mathbb{R}$ and investigate the basic properties and some examples of it. In section 4, using the concepts of the conditional Wiener integral 21, we define a measure-valued measure $V_{\varphi}$ on $C[0, t]$, associated with a measure $\omega_{\varphi}$ and investigate its basic properties. In section 5, we establish the Feynman-Kac formula with respect to $V_{\varphi}$. In the last section, we prove that our result in section 5 satisfies a suitable Volterra integral equation.

\section{Preliminaries}

In this section, we introduce some notation, definitions and facts which are needed to understand the subsequent sections.

(A) Let $\mathbb{R}$ be the real number system and let $\mathbb{C}$ be the complex number system. For a natural number $n$, let $\mathbb{R}^{n}$ be the $n$-times product space of $\mathbb{R}$. Let $\mathcal{B}(\mathbb{R})$ be the set of all Borel measurable subsets of $\mathbb{R}$ and let $m_{L}$ be the Lebesgue measure on the measurable space $(\mathbb{R}, \mathcal{B}(\mathbb{R}))$. Let $\alpha_{1}=1, \alpha_{2}=-1, \alpha_{3}=i$ and $\alpha_{4}=-i$.

(B) For a positive real number $t$, let $C[0, t]$ be the space of all real-valued continuous functions on a closed bounded interval $[0, t]$ with the supremum norm $\|\cdot\|_{\infty}$. By the Stone-Weierstrass theorem,

$$
\left(C[0, t],\|\cdot\|_{\infty}\right) \text { is a real separable Banach space. }
$$

Let $\mathcal{M}(\mathbb{R})$ be the space of all finite complex-valued countably additive measures on $(\mathbb{R}, \mathcal{B}(\mathbb{R}))$. For $p$ in $\mathbb{R}$, let $\delta_{p}$ be the Dirac measure concentrated at $p$ with total mass one. For $\mu$ in $\mathcal{M}(\mathbb{R})$ and for $E$ in $\mathcal{B}(\mathbb{R})$, the total variation $|\mu|(E)$ on $E$ is defined by

$$
|\mu|(E)=\sup \sum_{i=1}^{n}\left|\mu\left(E_{i}\right)\right|
$$

where the supremum is taken over all finite sequences $\left\langle E_{i}\right\rangle$ of disjoint sets in $\mathcal{B}(\mathbb{R})$. Then $|\mu|$ is in $\mathcal{M}(\mathbb{R})$ and, by the Jordan decomposition theorem [7, (19.13) Theorem, p. 307], there are unique nonnegative measures $\mu_{j}(j=1,2,3,4)$ in $\mathcal{M}(\mathbb{R})$ such that

$$
\mu=\sum_{j=1}^{4} \alpha_{j} \mu_{j} .
$$

By [3, Theorem 4.1.7, p. 128] $(\mathcal{M}(\mathbb{R}),|\cdot|(\mathbb{R}))$ is a complex Banach space. Let $\mathcal{R} \mathcal{M}(\mathbb{R})$ be the space of all finite complex-valued measures $\mu$ on $(\mathbb{R}, \mathcal{B}(\mathbb{R}))$ that are absolutely continuous with respect to $m_{L}$, that is, the Radon-Nikodym derivative $\frac{d|\mu|}{d m_{L}}$ exists.

(C) Let $(X, \mathcal{B}, \mu)$ be a measure space. For a positive real number $p$, let $\mathcal{L}^{p}(X, \mu)$ be the space of complex-valued $\mu$-measurable functions $f$ on $X$ such that $|f|^{p}$ is $|\mu|^{-}$ integrable. Let $\mathcal{L}^{\infty}(X, \mu)$ be the space of complex-valued $\mu$-measurable functions $f$ on $X$ that are $|\mu|$-essentially bounded. The elements of $\mathcal{L}^{p}(X, \mu)$ and $\mathcal{L}^{\infty}(X, \mu)$ are equivalence classes of functions in $\mathcal{L}^{p}(X, \mu)$ and $\mathcal{L}^{\infty}(X, \mu)$, respectively, with $f_{1}$ and $f_{2}$ said to be equivalent if they are equal $|\mu|$-a.e. Since $\mathcal{R M}(\mathbb{R})$ is isomorphic to $L^{1}\left(\mathbb{R}, m_{L}\right), \mathcal{R} \mathcal{M}(\mathbb{R})$ is a Banach space and the dual space $\mathcal{R} \mathcal{M}(\mathbb{R})^{*}$ of $\mathcal{R} \mathcal{M}(\mathbb{R})$ is 
isomorphic to $L^{\infty}\left(\mathbb{R}, m_{L}\right)$. For $x^{*}$ in $\mathcal{R} \mathcal{M}(\mathbb{R})^{*}$, there is a function $\theta$ in $L^{\infty}\left(\mathbb{R}, m_{L}\right)$ such that $x^{*}(\mu)=\int_{\mathbb{R}} \theta(s) d \mu(s)$ for $\mu$ in $\mathcal{R} \mathcal{M}(\mathbb{R})$.

Let $\mathbb{B}$ be a complex Banach space and let $\mathbb{B}^{*}$ be the dual space of $\mathbb{B}$. For a $\mathbb{B}$-valued countably additive measure $\nu$ on $(X, \mathcal{B})$ and for $E$ in $\mathcal{B}$, the semivariation $\|\nu\|(E)$ of $\nu$ on $E$ is given by

$$
\|\nu\|(E)=\sup \left\{\left|x^{*} \nu\right|(E) \mid x^{*} \text { is in } \mathbb{B}^{*} \text { and }\left\|x^{*}\right\|_{\mathbb{B}^{*}} \leq 1\right\}
$$

where $\left|x^{*} \nu\right|(E)$ is the total variation on $E$ of the complex-valued measure $x^{*} \nu$.

(D) Let $\mathbb{B}$ be a complex Banach space and let $(X, \mathcal{B}, \mu)$ be a complex measure space. A function $f: X \rightarrow \mathbb{B}$ is called $\mu$-measurable if there exists a sequence $\left\langle f_{n}\right\rangle$ of $\mathbb{B}$-valued simple functions with

$$
\lim _{n \rightarrow \infty}\left\|f_{n}-f\right\|_{\mathbb{B}}=0 \quad|\mu| \text {-a.e. }
$$

A function $f$ is called $\mu$-weakly measurable if $x^{*} f$ is $\mu$-measurable for each $x^{*}$ in $\mathbb{B}^{*}$, the dual space of $\mathbb{B}$. By Pettis' measurability theorem [4, Theorem 2, p. 42],

$$
\begin{aligned}
& f \text { is } \mu \text {-measurable if and only if } f \text { is }|\mu| \text {-essentially, } \\
& \text { separably valued and } f \text { is } \mu \text {-weakly measurable. }
\end{aligned}
$$

We say that $f$ is $\mu$-Bochner integrable if there exists a sequence $\left\langle f_{n}\right\rangle$ of $\mathbb{B}$-valued simple functions such that $\left\langle f_{n}\right\rangle$ converges to $f$ in the norm sense in $\mathbb{B}$ for $|\mu|$-a.e. and $\lim _{n \rightarrow \infty} \int_{X}\left\|f(t)-f_{n}(t)\right\|_{\mathbb{B}} d|\mu|(t)=0$. In this case, $(B o)-\int_{X} f(t) d \mu(t)$ is defined by

$$
(B o)-\int_{X} f(t) d \mu(t)=\lim _{n \rightarrow \infty} \int_{X} f_{n}(t) d \mu(t)
$$

where the limit means the limit in the norm sense. By [4 Theorem 2, p. 45],

$$
f \text { is } \mu \text {-Bochner integrable if and only if } \int_{X}\|f(t)\|_{\mathbb{B}} d|\mu|(t) \text { is finite. }
$$

By [23, Corollary 2, p. 134],

if $U$ is a bounded linear operator on $\mathbb{B}$ into a Banach space $\mathbb{B}_{1}$ and $f$ is a $\mathbb{B}$-valued $\mu$-Bochner integrable function, then $U f$ is a $\mathbb{B}_{1}$-valued $\mu$-Bochner integrable function, and

$$
(B o)-\int_{X}(U f)(t) d \mu(t)=U\left((B o)-\int_{X} f(t) d \mu(t)\right) .
$$

Theorem 2.1. Let $(X, \mathcal{B}, \mu)$ be a complex measure space and let $f: X \rightarrow \mathcal{M}(\mathbb{R})$ be a $\mu$-Bochner integrable function. Then for $E$ in $\mathcal{B}(\mathbb{R}),[f(t)](E)$ is a complex-valued $\mu$-integrable function of $t$ and

$$
\left[(B o)-\int_{X} f(t) d \mu(t)\right](E)=\int_{X}[f(t)](E) d \mu(t) .
$$

Proof. For $B$ in $\mathcal{B}$ and for $m$ in $\mathcal{M}(\mathbb{R})$ we let $f(t)=\chi_{B}(t) m$ where $\chi_{B}(t)$ is a characteristic function associated with $B$. Then, for $E$ in $\mathcal{B}(\mathbb{R})$, trivially, $[f(t)](E)=$ 
$\chi_{B}(t) m(E)$ is $\mu$-integrable in $t$ and

$$
\begin{aligned}
& {\left[(B o)-\int_{X} f(t) d \mu(t)\right](E)} \\
& \quad=[\mu(B) m](E) \\
& \quad=\mu(B) m(E) \\
& \quad=\int_{X}[f(t)](E) d \mu(t) ;
\end{aligned}
$$

so this theorem holds whenever $f(t)=\chi_{B}(t) m$ and $B$ is in $\mathcal{B}$. By the basic properties of the Bochner integral and the Lebesgue integral, clearly this theorem holds whenever $f$ is a simple function. Now, we assume that $f$ is $\mu$-Bochner integrable. Then there is a sequence $\left\langle f_{n}\right\rangle$ of simple functions such that $\lim _{n \rightarrow \infty}\left|f_{n}(t)-f(t)\right|(\mathbb{R})=$ $0|\mu|$-a.e. $t$ and $\lim _{n \rightarrow \infty} \int_{X}\left|f_{n}(t)-f(t)\right|(\mathbb{R}) d|\mu|(t)=0$. Let $E$ be in $\mathcal{B}(\mathbb{R})$. Since $\left[f_{n}(t)\right](E)$ is simple for all natural numbers $n$ and $\left|\left[f_{n}(t)\right](E)-[f(t)](E)\right| \leq \mid f_{n}(t)-$ $f(t) \mid(\mathbb{R}) \rightarrow 0$ as $n \rightarrow+\infty,|\mu|$-a.e. $t,[f(t)](E)$ is a complex-valued $\mu$-measurable function. By $(2.8), \int_{X}|[f(t)](E)| d|\mu|(t) \leq \int_{X}|f(t)|(\mathbb{R}) d|\mu|(t)<+\infty$; so $[f(t)](E)$ is $|\mu|$-integrable. By [7, (19.16) Theorem, p. 311], $[f(t)](E)$ is $\mu$-integrable. Moreover,

$$
\begin{aligned}
& \left|\left[(B o)-\int_{X} f(t) d \mu(t)\right](E)-\int_{X}[f(t)](E) d \mu(t)\right| \\
& \quad \stackrel{(1)}{=} \lim _{n \rightarrow \infty}\left|\left[(B o)-\int_{X} f_{n}(t) d \mu(t)\right](E)-\int_{X}[f(t)](E) d \mu(t)\right| \\
& \quad \stackrel{(2)}{=} \lim _{n \rightarrow \infty}\left|\int_{X}\left[f_{n}(t)\right](E) d \mu(t)-\int_{X}[f(t)](E) d \mu(t)\right| \\
& \quad \stackrel{(3)}{\leq} \lim _{n \rightarrow \infty} \int_{X}\left|\left[f_{n}(t)\right](E)-[f(t)](E)\right| d|\mu|(t) \\
& \quad \stackrel{(4)}{\leq} \lim _{n \rightarrow \infty} \int_{X}\left|f_{n}(t)-f(t)\right|(\mathbb{R}) d|\mu|(t) \\
& \quad \stackrel{(5)}{=} 0 .
\end{aligned}
$$

By (2.7) and the continuity of the absolute value, we have Step (1). We proved already that this theorem holds for $\mathcal{M}(\mathbb{R})$-valued simple functions in (2.11); so Step (2) is true. Step (3) results from the basic properties of the Lebesgue integral. By the definition (2.2) of the total variation for a measure $\mu$ on $(\mathbb{R}, \mathcal{B}(\mathbb{R}))$, we obtain Step (4). Step (5) follows from the assumptions for a sequence $\left\langle f_{n}\right\rangle$. Thus, we have $\left[(B o)-\int_{X} f(t) d \mu(t)\right](E)=\int_{X}[f(t)](E) d \mu(t)$.

Remark 2.2. Consider a function $H$ on $[0,1] \times[0,1]$ defined by $H(x, y)=\chi_{[0, x]}(y)$. Then $H$ is $\left(m_{L} \times m_{L}\right)$-integrable on $[0,1] \times[0,1]$; so by the Fubini theorem, $H(x, y)$ is an $m_{L}$-integrable function of $x$ for all $y$ and $H(x, \cdot)$ is in $L^{\infty}\left([0,1], m_{L}\right)$ for all $x$ in $[0,1]$. But $H(x, \cdot)$ has no essentially separable range; so $H(x, \cdot)$ is not $m_{L}$-Bochner integrable. Hence, in general, the equality $(2.10)$ is not true in the theory of the Bochner integral.

(E) Let $\mathbb{B}$ be a complex Banach space and let $(Y, \mathcal{C}, \nu)$ be a $\mathbb{B}$-valued measure space. Let $g$ be a complex-valued $\|\nu\|$-measurable function on $Y$, that is, there 
WIENER MEASURE AND THE MEASURE-VALUED FEYNMAN-KAC FORMULA 4925

exists a sequence $\left\langle g_{n}\right\rangle$ of complex-valued simple functions with $\lim _{n \rightarrow \infty}\left|g_{n}-g\right|=0$ $\|\nu\|$-a.e. We say that $g$ is $\nu$-Bartle integrable if there exists a sequence $\left\langle g_{n}\right\rangle$ of simple functions such that $\left\langle g_{n}\right\rangle$ converges to $g\|\nu\|$-a.e. and the sequence $\left\langle\int_{Y} g_{n}(s) d \nu(s)\right\rangle$ is Cauchy in the norm sense. In this case, $(B a)-\int_{Y} g(s) d \nu(s)$ is defined by

$$
(B a)-\int_{Y} g(s) d \nu(s)=\lim _{n \rightarrow \infty} \int_{Y} g_{n}(s) d \nu(s)
$$

where the limit means the limit in the norm sense. By [5 Theorem 8, p. 323],

if $f$ is a $\nu$-measurable function that is $\|\nu\|-$ essentially bounded, then $f$ is $\nu$-Bartle integrable and

$$
\left\|(B a)-\int_{Y} f(s) d \nu(s)\right\|_{\mathbb{B}} \leq(\|\nu\|-\operatorname{ess} \sup |f(s)|)\|\nu\|(Y) .
$$

By [15, Theorem 2.4, p. 162],

$g$ is $\nu$-Bartle integrable if and only if for each $x^{*}$ in

$\mathbb{B}^{*}, g$ is $x^{*} \nu$-integrable and for each $E$ in $\mathcal{C}$, there is an

element $(B a)-\int_{E} g(s) d \nu(s)$ in $\mathbb{B}$ such that $x^{*}\left[(B a)-\int_{E} g(s) d \nu(s)\right]$

$=\int_{E} g(s) d x^{*} \nu(s)$ for $x^{*}$ in $\mathbb{B}^{*}$.

By [5. Theorem 8, p. 324],

if $U$ is a bounded linear operator from $\mathbb{B}$ into a Banach space $\mathbb{B}_{1}$ and $g$ is $\nu$-Bartle integrable, then $g$ is $U \nu$-Bartle integrable. In this case,

$$
U\left[(B a)-\int_{Y} g(s) d \nu(s)\right]=(B a)-\int_{Y} g(s) d U \nu(s) .
$$

By [5, Theorem 10, p. 328],

if $\left\langle f_{n}\right\rangle$ is a sequence of $\nu$-Bartle integrable functions that converges $\|\nu\|$-a.e. to $f$ and if $g$ is a $\nu$-Bartle integrable function such that $\left|f_{n}(s)\right| \leq g(s)\|\nu\|$-a.e. $s$ for all natural numbers $n$, then $f$ is $\nu$-Bartle integrable and for $E$ in $\mathcal{C},(B a)-\int_{E} f(s) d \nu(s)=\lim _{n \rightarrow \infty}(B a)-\int_{E} f_{n}(s) d \nu(s)$.

$(\mathbf{F})$ Let $\mathbb{B}$ be a complex Banach space. Let $(X, \mathcal{B})$ and $(Y, \mathcal{C})$ be two measurable spaces and let $\mathcal{B} \otimes \mathcal{C}$ be the $\sigma$-algebra of sets in the space $X \times Y$ generated by the family of rectangles $E \times F$ for all $E$ in $\mathcal{B}$ and $F$ in $\mathcal{C}$. Let $\mu$ be a complex-valued measure on $(X, \mathcal{B})$ and let $\nu$ be a $\mathbb{B}$-valued measure on $(Y, \mathcal{C})$. For $G$ in $\mathcal{B} \otimes \mathcal{C}$, we let

$$
(\mu \times \nu)(G)=(B a)-\int_{Y}\left[\int_{X} \chi_{G}(u, v) d \mu(u)\right] d \nu(v) .
$$


By [10, Proposition 2, p. 169], using the dominated convergence theorem in [11. Theorem 2, p. 30], Kluvanek proved that $\mu \times \nu$ is a $\mathbb{B}$-valued measure on $\mathcal{B} \otimes \mathcal{C}$ and for $G$ in $\mathcal{B} \otimes \mathcal{C}$, the following holds:

$$
\begin{aligned}
(\mu & \times \nu)(G) \\
& =(B a)-\int_{Y}\left[\int_{X} \chi_{G}(u, v) d \mu(u)\right] d \nu(v) \\
& =(B o)-\int_{X}\left[(B a)-\int_{Y} \chi_{G}(u, v) d \nu(v)\right] d \mu(u) .
\end{aligned}
$$

Moreover, in [10, Proposition 3, p. 170], he showed that

$$
x^{*}(\mu \times \nu)=\mu \times\left(x^{*} \nu\right)
$$

for all $x^{*}$ in $\mathbb{B}^{*}$.

When both measures $\mu$ and $\nu$ are complex-valued, a sufficient condition for validity of the Fubini theorem is the integrability of the function with respect to $\mu \times \nu$. But, if $\nu$ is a vector measure, then the integrability of the function with respect to $\mu \times \nu$ is no longer a sufficient condition for the validity of the Fubini theorem. Indeed, we can find a counterexample for this fact in 10, Example, p. 170].

Theorem 2.3. Let $\mathbb{B}$ be a separable complex Banach space, $(X, \mathcal{B}, \mu)$ a complexvalued measure space and let $(Y, \mathcal{C}, \nu)$ be a $\mathbb{B}$-valued measure space. Let $f: X \times Y \rightarrow$ $\mathbb{C}$ be $(\mathcal{B} \otimes \mathcal{C})$-measurable and $(\mu \times \nu)$-Bartle integrable. Then

$$
\begin{gathered}
\text { for }\|\nu\| \text {-a.e. } v, f(u, v) \text { is a } \mu \text {-integrable function of } u, \\
\int_{X} f(u, v) d \mu(u) \text { is } \nu \text {-Bartle integrable, and } \\
(B a)-\int_{X \times Y} f(u, v) d \mu \times \nu(u, v) \\
=(B a)-\int_{Y}\left[\int_{X} f(u, v) d \mu(u)\right] d \nu(v) .
\end{gathered}
$$

Moreover, if for $|\mu|$-a.e. $u, f(u, v)$ is a $\nu$-Bartle integrable function of $v$ and $(B a)-$ $\int_{Y} f(u, v) d \nu(v)$ is $\mu$-Bochner integrable, then

$$
\begin{aligned}
(B a) & -\int_{X \times Y} f(u, v) d \mu \times \nu(u, v) \\
& =(B o)-\int_{X}\left[(B a)-\int_{Y} f(u, v) d \nu(v)\right] d \mu(u) \\
& =(B a)-\int_{Y}\left[\int_{X} f(u, v) d \mu(u)\right] d \nu(v) .
\end{aligned}
$$

Proof. In [10, Proposition 4, p. 171], one can find that the facts (2.21), (2.22) and (2.23) hold. By the classical Fubini theorem, for $x^{*}$ in $\mathbb{B}^{*}$,

$$
x^{*}\left[(B a)-\int_{Y} f(u, v) d \nu(v)\right]=\int_{Y} f(u, v) d x^{*} \nu(v)
$$


is a $|\mu|$-measurable function of $u$. Since $\mathbb{B}$ is separable, by condition (2.6), (Ba)$\int_{Y} f(u, v) d \nu(v)$ is a $|\mu|$-measurable function of $u$. For $x^{*}$ in $\mathbb{B}^{*}$,

$$
\begin{aligned}
x^{*}[ & \left.(B a)-\int_{X \times Y} f(u, v) d \mu \times \nu(u, v)\right] \\
& \stackrel{(1)}{=} \int_{X \times Y} f(u, v) d\left[x^{*}(\mu \times \nu)\right](u, v) \\
& \stackrel{(2)}{=} \int_{X \times Y} f(u, v) d\left[\mu \times\left(x^{*} \nu\right)\right](u, v) \\
& \stackrel{(3)}{=} \int_{X}\left[\int_{Y} f(u, v) d\left(x^{*} \nu\right)(v)\right] d \mu(u) \\
& \stackrel{(4)}{=} \int_{X} x^{*}\left[(B a)-\int_{Y} f(u, v) d \nu(v)\right] d \mu(u) \\
& \stackrel{(5)}{=} x^{*}\left[(B o)-\int_{X}\left\{(B a)-\int_{Y} f(u, v) d \nu(v)\right\} d \mu(u)\right] .
\end{aligned}
$$

Step (1) results from (2.15). Step (2) follows from (2.20). By the classical Fubini theorem, we have Step (3). From (2.16), we obtain Step (4). By (2.9), we have Step (5).

Using the Hahn-Banach theorem, one can show

$$
\begin{aligned}
(B a) & -\int_{X \times Y} f(u, v) d \mu \times \nu(u, v) \\
& =(B o)-\int_{X}\left[(B a)-\int_{Y} f(u, v) d \nu(v)\right] d \mu(u),
\end{aligned}
$$

as desired.

(G) Let $\varphi$ be in $\mathcal{M}(\mathbb{R})$ and $\eta$ be a complex-valued Borel measure on $[0, t]$. A complex-valued Borel measurable function $\theta$ on $[0, t] \times \mathbb{R}$ is said to belong to $L_{\varphi ; \infty, 1 ; \eta}$ (or $L_{\varphi ; \infty, 1 ; \eta}^{t}$ ) if

$$
\|\theta\|_{\varphi ; \infty, 1 ; \eta}=\int_{[0, t]}\|\theta(s, \cdot)\|_{\varphi ; \infty} d|\eta|(s)
$$

is finite where $\|\theta(0, \cdot)\|_{\varphi ; \infty}$ is $\inf \{\lambda>0|| \varphi \mid(\{\xi$ in $\mathbb{R}|| \theta(0, \xi) \mid>\lambda\})=0\}$ and $\|\theta(s, \cdot)\|_{\varphi ; \infty}$ is $\inf \left\{\lambda>0 \mid m_{L}(\{\xi\right.$ in $\left.\mathbb{R}|| \theta(s, \xi) \mid>\lambda\})=0\right\}$ for $0<s \leq t$. If $\theta$ is bounded Borel measurable, then $\theta$ is in $L_{\varphi ; \infty, 1 ; \eta}$.

(H) For $\theta$ in $L^{\infty}\left(\mathbb{R}, m_{L}\right)$, we consider an operator $M_{\theta}$ from $\mathcal{R} \mathcal{M}(\mathbb{R})$ into itself such that

$$
\left[M_{\theta}(\mu)\right](E)=\int_{E} \frac{d \mu}{d m_{L}}(\xi) \theta(\xi) d m_{L}(\xi)
$$

for $E$ in $\mathcal{B}(\mathbb{R})$ and for $\mu$ in $\mathcal{R M}(\mathbb{R})$. Then

$$
\frac{d M_{\theta}(\mu)}{d m_{L}}(\xi)=\frac{d \mu}{d m_{L}}(\xi) \theta(\xi) ;
$$

so $M_{\theta}$ is well-defined. Since $\left|M_{\theta}(\mu)\right|(\mathbb{R}) \leq \int_{\mathbb{R}}\left|\frac{d \mu}{d m_{L}}(\xi)\right||\theta(\xi)| d m_{L}(\xi) \leq\|\theta\|_{\infty}$ $|\mu|(\mathbb{R}), M_{\theta}$ is a bounded linear operator. 
For $s>0$, we let

$$
P_{s}(E)=\int_{E} \frac{1}{\sqrt{2 \pi s}} \exp \left\{-\frac{u^{2}}{2 s}\right\} d m_{L}(u)
$$

for $E$ in $\mathcal{B}(\mathbb{R})$.

For $s>0$, we consider an operator $S_{s}$ from $\mathcal{R} \mathcal{M}(\mathbb{R})$ into itself such that

$$
\begin{aligned}
& {\left[S_{s}(\mu)\right](E)=\left(\mu * P_{s}\right)(E)} \\
& \quad=\frac{1}{\sqrt{2 \pi s}} \int_{\mathbb{R}}\left[\int_{E} \exp \left\{-\frac{(u-v)^{2}}{2 s}\right\} d m_{L}(u)\right] d \mu(v) .
\end{aligned}
$$

Then $\frac{d S_{s}(\mu)}{d m_{L}}(\xi)=\frac{1}{\sqrt{2 \pi s}} \int_{\mathbb{R}} \exp \left\{-\frac{(\xi-v)^{2}}{2 s}\right\} d \mu(v)$; so $S_{s}$ is well-defined. It is not hard to show that $S_{s}$ is a bounded linear operator and the operator norm $\left\|S_{s}\right\|$ of $S_{s}$ is less than or equal to one.

Let $s_{1}$ and $s_{2}$ be two positive real numbers. Then by the Chapman-Kolmogorov equation in [8. Proposition 3.2.3, p. 37] and the classical Fubini theorem, we have

$$
S_{s_{1}} \circ S_{s_{2}}=S_{s_{1}+s_{2}} .
$$

For $s>0$, for $\varphi$ in $\mathcal{M}(\mathbb{R})$, for a Borel measurable $|\varphi|$-essentially bounded function $\theta$ on $(\mathbb{R}, \mathcal{B}(\mathbb{R}))$ and for $E$ in $\mathcal{B}(\mathbb{R})$, we let

$$
[T(s, \varphi, \theta)](E)=\frac{1}{\sqrt{2 \pi s}} \int_{\mathbb{R}}\left[\int_{E} \theta(v) \exp \left\{-\frac{(u-v)^{2}}{2 s}\right\} d m_{L}(u)\right] d \varphi(v) .
$$

Then $T(s, \varphi, \theta)$ is in $\mathcal{R} \mathcal{M}(\mathbb{R})$ and

$$
\frac{d T(s, \varphi, \theta)}{d m_{L}}(u)=\frac{1}{\sqrt{2 \pi s}} \int_{\mathbb{R}} \theta(v) \exp \left\{-\frac{(u-v)^{2}}{2 s}\right\} d \varphi(v) .
$$

\section{The complex-Valued analogue of the Wiener measure $\omega_{\varphi}$}

In this section, we will introduce a complex-valued analogue of the Wiener measure $\omega_{\varphi}$ on $C[0, t]$ and we will give some examples of it.

Let $t$ be a positive real number and $n$ a nonnegative integer. For $\vec{t}=\left(t_{0}, t_{1}, \cdots\right.$, $\left.t_{n}\right)$ with $0=t_{0}<t_{1}<\cdots<t_{n} \leq t$, let $J_{\vec{t}}: C[0, t] \rightarrow \mathbb{R}^{n+1}$ be a function with

$$
J_{\vec{t}}(x)=\left(x\left(t_{0}\right), x\left(t_{1}\right), \cdots, x\left(t_{n}\right)\right) .
$$

For $B_{j}(j=0,1,2, \cdots, n)$ in $\mathcal{B}(\mathbb{R})$, the subset $J_{\vec{t}}^{-1}\left(\prod_{j=0}^{n} B_{j}\right)$ of $C[0, t]$ is called an interval. Let $\mathcal{I}$ be the set of all intervals. For a nonnegative finite Borel measure $\varphi$ on $(\mathbb{R}, \mathcal{B}(\mathbb{R}))$, we let

$$
\begin{aligned}
& m_{\varphi}\left(J_{\vec{t}}^{-1}\left(\prod_{j=0=0}^{n} B_{j}\right)\right) \\
& \quad=\int_{B_{0}}\left[\int_{\prod_{j=1}^{n} B_{j}} W\left(n+1 ; \vec{t} ; u_{0}, u_{1}, \cdots, u_{n}\right) d \prod_{j=1}^{n} m_{L}\left(u_{1}, \cdots, u_{n}\right)\right] d \varphi\left(u_{0}\right)
\end{aligned}
$$


where

$$
\begin{aligned}
W\left(n+1 ; \overrightarrow{t ;} u_{0}, u_{1}, \cdots, u_{n}\right) \\
\quad=\left(\prod_{j=1}^{n} \frac{1}{\sqrt{2 \pi\left(t_{j}-t_{j-1}\right)}}\right) \exp \left\{-\frac{1}{2} \sum_{j=1}^{n} \frac{\left(u_{j}-u_{j-1}\right)^{2}}{t_{j}-t_{j-1}}\right\} .
\end{aligned}
$$

By [18, Theorem 5.1, p. 144] and [18, Theorem 2.1, p. 212], $\mathcal{B}(C[0, t])$, the set of all Borel subsets in $C[0, t]$, coincides with the smallest $\sigma$-algebra generated by $\mathcal{I}$ and there exists a unique positive measure $\omega_{\varphi}$ on $(C[0, t], \mathcal{B}(C[0, t]))$ such that $\omega_{\varphi}(I)=m_{\varphi}(I)$ for all $I$ in $\mathcal{I}$.

For $\varphi$ in $\mathcal{M}(\mathbb{R})$ with the Jordan decomposition $\varphi=\sum_{j=1}^{4} \alpha_{j} \varphi_{j}$, let $\omega_{\varphi}=$ $\sum_{j=1}^{4} \alpha_{j} \omega_{\varphi_{j}}$. We say that $\omega_{\varphi}$ is the complex-valued analogue of the Wiener measure on $(C[0, t], \mathcal{B}(C[0, t]))$, associated with $\varphi$. If $\varphi$ is a Dirac measure $\delta_{0}$ at the origin in $\mathbb{R}$, then $\omega_{\varphi}$ is the classical Wiener measure.

By the change of variables formula, we can easily prove the following theorem.

Theorem 3.1 (The Wiener integration formula). If $f: \mathbb{R}^{n+1} \rightarrow \mathbb{C}$ is a Borel measurable function, then the following equality holds:

$$
\begin{aligned}
\int_{C[0, t]} & f\left(x\left(t_{0}\right), x\left(t_{1}\right), \cdots, x\left(t_{n}\right)\right) d \omega_{\varphi}(x) \\
& \stackrel{*}{=} \int_{\mathbb{R}^{n+1}} f\left(u_{0}, u_{1}, \cdots, u_{n}\right) W\left(n+1 ; \vec{t} ; u_{0}, u_{1}, \cdots, u_{n}\right) \\
& d\left(\prod_{j=1}^{n} m_{L} \times \varphi\right)\left(\left(u_{1}, u_{2}, \cdots, u_{n}\right), u_{0}\right)
\end{aligned}
$$

where $\stackrel{*}{=}$ means that if one side exists, then both sides exist and the two values are equal.

Remark 3.2. Let $\varphi$ be in $\mathcal{M}(\mathbb{R})$.

(1) It is not hard to show that $\omega_{\varphi}$ has no atoms.

(2) $\omega_{\varphi}(C[0, t])=\varphi(\mathbb{R})$.

(3) Let $J_{t}: C[0, t] \rightarrow \mathbb{C}$ be a function with $J_{t}(x)=x(t)$. Then for $E$ in $\mathcal{B}(\mathbb{R})$, $\omega_{\varphi}\left(J_{t}^{-1}(E)\right)=\left[S_{t}(\varphi)\right](E)$.

Example 3.3. Let $\varphi$ be in $\mathcal{M}(\mathbb{R})$.

(1) Let $I=\{x$ in $C[0, t] \mid x(0)$ is in $B\}$ where $B$ is in $\mathcal{B}(\mathbb{R})$. Then $\omega_{\varphi}(I)=$ $\varphi(B)$.

(2) Suppose that $f(u)=u$ is $\varphi$-integrable. Then for $0 \leq s \leq t$,

$$
\int_{C[0, t]} x(s) d \omega_{\varphi}(x)=\int_{\mathbb{R}} u d \varphi(u) .
$$

If $\varphi=\delta_{p}$, then $\int_{C[0, t]} x(s) d \omega_{\varphi}(x)=p$; and if $\varphi$ has a normal distribution with mean $\alpha$ and variation $\sigma^{2}$, then $\int_{C[0, t]} x(s) d \omega_{\varphi}(x)=\alpha$.

(3) Suppose that $g(u)=u^{2}$ is $\varphi$-integrable. Then for $0 \leq s \leq t$,

$$
\int_{C[0, t]} x(s)^{2} d \omega_{\varphi}(x)=\int_{\mathbb{R}} u^{2} d \varphi(u)+s \varphi(\mathbb{R}) .
$$


If $\varphi=\delta_{p}$, then $\int_{C[0, t]} x(s)^{2} d \omega_{\varphi}(x)=p^{2}+s$ and if $\varphi$ has a normal distribution with mean $\alpha$ and variance $\sigma^{2}$, then

$$
\int_{C[0, t]} x(s)^{2} d \omega_{\varphi}(x)=\alpha^{2}+\sigma^{2}+s .
$$

(4) Let $\mathcal{F}(\varphi)$ be the Fourier transform of a measure $\varphi$, that is, $[\mathcal{F}(\varphi)](\xi)=$ $\int_{\mathbb{R}} \exp \{i \xi u\} d \varphi(u)$. Then for $0 \leq s \leq t$,

$$
\int_{C[0, t]} \exp \{i \xi x(s)\} d \omega_{\varphi}(x)=\exp \left\{-\frac{s \xi^{2}}{2}\right\}[\mathcal{F}(\varphi)](\xi) .
$$

If $\varphi=\delta_{p}$, then $\int_{C[0, t]} \exp \{i \xi x(s)\} d \omega_{\varphi}(x)=\exp \left\{-\frac{s \xi^{2}}{2}+i p \xi\right\}$ and if $\varphi$ has a normal distribution with mean $\alpha$ and variance $\sigma^{2}$ then

$$
\int_{C[0, t]} \exp \{i \xi x(s)\} d \omega_{\varphi}(x)=\exp \left\{-\frac{\left(s+\sigma^{2}\right) \xi^{2}}{2}+i \alpha \xi\right\} .
$$

Let $0<s \leq t$ be given and let $J_{s}: C[0, t] \rightarrow \mathbb{R}$ be a function with $J_{s}(x)=x(s)$. We assume that $\left\langle\varphi_{n}\right\rangle$ converges to $\varphi$ weakly. By a similar calculation as in this example, since $\left\langle\mathcal{F}\left(\varphi_{n}\right)\right\rangle$ converges to $\mathcal{F}(\varphi)$ pointwise, $\left\langle\mathcal{F}\left(\omega_{\varphi_{n}}\left(J_{s}^{-1}(\cdot)\right)\right)\right\rangle$ converges to $\mathcal{F}\left(\omega_{\varphi}\left(J_{s}^{-1}(\cdot)\right)\right)$ pointwise; so by the continuity theorem in [1, Theorem 12-5A, p. $273],\left\langle\omega_{\varphi_{n}}\left(J_{s}^{-1}(\cdot)\right)\right\rangle$ converges to $\omega_{\varphi}\left(J_{s}^{-1}(\cdot)\right)$ weakly.

(5) We assume that $k(u)=u^{2}$ is $\varphi$-integrable. For $0 \leq s_{1}, s_{2} \leq t$,

$$
\int_{C[0, t]} x\left(s_{1}\right) x\left(s_{2}\right) d \omega_{\varphi}(x)=\left(\min \left\{s_{1}, s_{2}\right\}\right) \varphi(\mathbb{R})+\int_{\mathbb{R}} u^{2} d \varphi(u) .
$$

If $\varphi=\delta_{p}$, then $\int_{C[0, t]} x\left(s_{1}\right) x\left(s_{2}\right) d \omega_{\varphi}(x)=\min \left\{s_{1}, s_{2}\right\}+p^{2}$ and if $\varphi$ has a normal distribution with mean $\alpha$ and variance $\sigma^{2}$, then

$$
\int_{C[0, t]} x\left(s_{1}\right) x\left(s_{2}\right) d \omega_{\varphi}(x)=\min \left\{s_{1}, s_{2}\right\}+\alpha^{2}+\sigma^{2} .
$$

(6) For $0 \leq s_{1}<s_{2} \leq s_{3}<s_{4} \leq t$ and for $\alpha, \beta$ in $\mathbb{R}$, using the change of variable formula, we have

$$
\begin{gathered}
\omega_{\varphi}\left(\left\{x \text { in } C[0, t] \mid x\left(s_{2}\right)-x\left(s_{1}\right) \leq \alpha \text { and } x\left(s_{4}\right)-x\left(s_{3}\right) \leq \beta\right\}\right) \\
=\varphi(\mathbb{R}) \omega_{\varphi}\left(\left\{x \text { in } C[0, t] \mid x\left(s_{2}\right)-x\left(s_{1}\right) \leq \alpha\right\}\right) \\
\cdot \omega_{\varphi}\left(\left\{x \text { in } C[0, t] \mid x\left(s_{4}\right)-x\left(s_{3}\right) \leq \beta\right\}\right) .
\end{gathered}
$$

Hence, if $\varphi$ is a probability measure, then $x\left(s_{2}\right)-x\left(s_{1}\right)$ and $x\left(s_{4}\right)-x\left(s_{3}\right)$ are independent.

Theorem 3.4. For $\varphi$ in $\mathcal{M}(\mathbb{R}),\left|\omega_{\varphi}\right|=\omega_{|\varphi|}$ on $(C[0, t], \mathcal{B}(C[0, t]))$.

Proof. By [19, Theorem 6.12, p. 124], there exists a measurable function $h$ such that $|h(u)|=1$ for all $u$ in $\mathbb{R}$ and $d \varphi=h d|\varphi|$. For $\vec{t}=\left(t_{0}, t_{1}, \cdots, t_{n}\right)$ with $0=t_{0}<t_{1}, \cdots, t_{n} \leq t$ and for $B_{j}(j=0,1, \cdots, n)$ in $\mathcal{B}(\mathbb{R})$, by [19] Theorem 6.13, 
WIENER MEASURE AND THE MEASURE-VALUED FEYNMAN-KAC FORMULA 4931

p. 125] and (3.3),

$$
\begin{aligned}
& \omega_{\varphi}\left(J_{\vec{t}}^{-1}\left(\prod_{j=0}^{n} B_{j}\right)\right) \\
& \quad=\int_{\mathbb{R}}\left[\int_{\mathbb{R}^{n}} W\left(n+1 ; \vec{t} ; u_{0}, u_{1}, \cdots, u_{n}\right) d\left(\prod_{j=1}^{n} m_{L}\right)\left(u_{1}, \cdots, u_{n}\right)\right] d \varphi\left(u_{0}\right) \\
& \quad=\int_{\mathbb{R}} h\left(u_{0}\right)\left[\int_{\mathbb{R}^{n}} W\left(n+1 ; \vec{t} ; u_{0}, u_{1}, \cdots, u_{n}\right) d\left(\prod_{j=1}^{n} m_{L}\right)\left(u_{1}, \cdots, u_{n}\right)\right] d|\varphi|\left(u_{0}\right)
\end{aligned}
$$

and so

$$
\begin{aligned}
\left|\omega_{\varphi}\right| & \left(J_{\vec{t}}^{-1}\left(\prod_{j=0}^{n} B_{j}\right)\right) \\
& =\int_{\mathbb{R}}\left[\int_{\mathbb{R}^{n}} W\left(n+1 ; \vec{t} ; u_{0}, u_{1}, \cdots, u_{n}\right) d\left(\prod_{j=1}^{n} m_{L}\right)\left(u_{1}, \cdots, u_{n}\right)\right] d|\varphi|\left(u_{0}\right) \\
& =\omega_{|\varphi|}\left(J_{\vec{t}}^{-1}\left(\prod_{j=0}^{n} B_{j}\right)\right)
\end{aligned}
$$

We consider a set $\mathcal{A}=\left\{E\right.$ in $\left.\mathcal{B}(C[0, t])|| \omega_{\varphi} \mid(E)=\omega_{|\varphi|}(E)\right\}$. Then, by the equality (3.5), we have $\mathcal{I} \subset \mathcal{A}$. Since $\left|\omega_{\varphi}\right|$ and $\omega_{|\varphi|}$ are both measures on $(C[0, t], \mathcal{B}(C[0, t]))$, by [18, Theorem 2.1, p. 212], $\left|\omega_{\varphi}\right|=\omega_{|\varphi|}$ on $\mathcal{B}(C[0, t])$.

4. The measure-valued measure $V_{\varphi}$ On $(C[0, t], \mathcal{B}(C[0, t]))$

In this section, we introduce a measure-valued measure $V_{\varphi}$ on $(C[0, t], \mathcal{B}(C[0, t]))$ from the concepts of an analogue of the Wiener measure $\omega_{\varphi}$ and investigate its basic properties.

Let $\varphi$ be in $\mathcal{M}(\mathbb{R})$ with the Jordan decomposition $\varphi=\sum_{j=1}^{4} \alpha_{j} \varphi_{j}$ and let $J_{t}: C[0, t] \rightarrow \mathbb{R}$ be a function defined by $J_{t}(x)=x(t)$.

For a measure $\mu$ in $\mathcal{M}(\mathbb{R})$, for $B$ in $\mathcal{B}(C[0, t])$ and for $E$ in $\mathcal{B}(\mathbb{R})$, let

$$
P_{\mu}(E)=\omega_{\mu}\left(J_{t}^{-1}(E)\right)
$$

and

$$
Q_{B, \mu}(E)=\omega_{\mu}\left(J_{t}^{-1}(E) \cap B\right) .
$$

Then both $P_{\mu}$ and $Q_{B, \mu}$ are measures on $(\mathbb{R}, \mathcal{B}(\mathbb{R}))$. By Theorem 3.4, we have

$$
\begin{aligned}
P_{\varphi} & =\sum_{j=1}^{4} \alpha_{j} P_{\varphi_{j}}, \\
Q_{B, \varphi} & =\sum_{j=1}^{4} \alpha_{j} Q_{B, \varphi_{j}},
\end{aligned}
$$


and

$$
\left|P_{\varphi}\right|=P_{|\varphi|} .
$$

For $j=1,2,3,4, Q_{B, \varphi_{j}}$ is absolutely continuous with respect to $P_{\varphi_{j}}$, and $P_{\varphi_{j}}$ is absolutely continuous with respect to $\left|P_{\varphi}\right|=P_{|\varphi|}$. Hence, for $j=1,2,3,4$, by the Radon-Nikodym theorem, there exists a measurable function $\widetilde{\psi}_{B, \varphi_{j}}$ such that $0 \leq \widetilde{\psi}_{B, \varphi_{j}} \leq 1$ and $Q_{B, \varphi_{j}}(E)=\int_{E} \widetilde{\psi}_{B, \varphi_{j}}(\xi) d P_{|\varphi|}(\xi)$ for $E$ in $\mathcal{B}(\mathbb{R})$. By 19 Theorem 6.12, p. 124], there is a measurable function $h$ such that $|h(\xi)|=1$ for all $\xi$ in $\mathbb{R}$ and $d P_{\varphi}=h d P_{|\varphi|}$; so $d P_{|\varphi|}=\bar{h} d P_{\varphi}$ where $\bar{h}$ is conjugate of $h$. Hence, we can write

$$
Q_{B, \varphi_{j}}(E)=\int_{E} \widetilde{\psi}_{B, \varphi_{j}}(\xi) \bar{h}(\xi) d P_{\varphi}(\xi)
$$

for $E$ in $\mathcal{B}(\mathbb{R})$ and for $j=1,2,3,4$. Thus,

$$
Q_{B, \varphi}(E)=\int_{E} \sum_{j=1}^{4} \alpha_{j} \widetilde{\psi}_{B, \varphi_{j}}(\xi) \bar{h}(\xi) d P_{\varphi}(\xi)
$$

For $B$ in $\mathcal{B}(C[0, t])$, we let

$$
\psi_{B, \varphi}=\left(\sum_{j=1}^{4} \alpha_{j} \widetilde{\psi}_{B, \varphi_{j}}\right) \bar{h} .
$$

By Theorem 3.1, the Radon-Nikodym derivative $\frac{d P_{\varphi}}{d m_{L}}$ exists and

$$
\frac{d P_{\varphi}}{d m_{L}}(\xi)=\int_{\mathbb{R}} \frac{1}{\sqrt{2 \pi t}} \exp \left\{-\frac{(\xi-u)^{2}}{2 t}\right\} d \varphi(u) ;
$$

so for $E$ in $\mathcal{B}(\mathbb{R})$,

$$
Q_{B, \varphi}(E)=\int_{\mathbb{R}}\left[\int_{E} \frac{1}{\sqrt{2 \pi t}} \psi_{B, \varphi}(\xi) \exp \left\{-\frac{(\xi-u)^{2}}{2 t}\right\} d m_{L}(\xi)\right] d \varphi(u) .
$$

For $B$ in $\mathcal{B}(C[0, t])$, we let

$$
\left[V_{\varphi}(B)\right](E)=Q_{B, \varphi}(E)
$$

for $E$ in $\mathcal{B}(\mathbb{R})$.

Remark 4.1. Let $\varphi$ be in $\mathcal{M}(\mathbb{R})$.

(1) From (4.2), for $E$ in $\mathcal{B}(\mathbb{R}), Q_{\emptyset, \varphi}(E)=0$, which implies that $\left[V_{\varphi}(\emptyset)\right](E)=0$ for $E$ in $\mathcal{B}(\mathbb{R})$.

(2) $P_{\varphi}=Q_{C[0, t], \varphi}$, and so, by (4.7), $\psi_{C[0, t], \varphi}=1 P_{|\varphi|}$-a.e. Hence, by (2.31), we have

$$
\left[V_{\varphi}(C[0, t])\right](E)=\left[S_{t}(\varphi)\right](E)
$$

for $E$ in $\mathcal{B}(\mathbb{R})$.

(3) Let $\varphi$ be in $\mathcal{M}(\mathbb{R})$ with the Jordan decomposition $\varphi=\sum_{j=1}^{4} \alpha_{j} \varphi_{j}$. Then from (4.4) and (4.11), we have $V_{\varphi}=\sum_{j=1}^{4} \alpha_{j} V_{\varphi_{j}}$. 
WIENER MEASURE AND THE MEASURE-VALUED FEYNMAN-KAC FORMULA 4933

(4) By Theorem 3.1 and equation (4.11), we know that $\left[V_{\alpha \varphi_{1}+\beta \varphi_{2}}(B)\right](E)=$ $\alpha\left[V_{\varphi_{1}}(B)\right](E)+\beta\left[V_{\varphi_{2}}(B)\right](E)$ for any $\alpha, \beta$ in $\mathbb{C}$, for any $\varphi_{1}, \varphi_{2}$ in $\mathcal{M}(\mathbb{R})$, for $B$ in $\mathcal{B}(C[0, t])$ and for $E$ in $\mathcal{B}(\mathbb{R})$. For $B$ in $\mathcal{B}(C[0, t])$, by $(\mathrm{C})$ in section 2 ,

$$
\begin{aligned}
& \left\|V_{\varphi}(B)\right\| \\
& \leq \sup _{\substack{\|\theta\|_{\infty} \leq 1 \\
\theta \in L^{\infty}\left(\mathbb{R}, m_{L}\right)}} \frac{1}{\sqrt{2 \pi t}} \int_{\mathbb{R}}\left[\int_{\mathbb{R}}\left|\psi_{B, \varphi}(\xi)\right| \exp \left\{-\frac{(\xi-u)^{2}}{2 t}\right\}|\theta(\xi)| d m_{L}(\xi)\right] d|\varphi|(u) \\
& \leq 4|\varphi|(\mathbb{R}) .
\end{aligned}
$$

Hence, letting $V(B): \mathcal{M}(\mathbb{R}) \rightarrow \mathcal{M}(\mathbb{R})$ with $[V(B)](\varphi)=V_{\varphi}(B), V(B)$ is a bounded linear operator on $\mathcal{M}(\mathbb{R})$.

Theorem 4.2. For $\varphi$ in $\mathcal{M}(\mathbb{R}), V_{\varphi}$ is a measure-valued measure on $(C[0, t]$, $\mathcal{B}(C[0, t]))$ in the total variation norm sense.

Proof. By Remark $4.1(1), V_{\varphi}(\emptyset)$ is a zero measure and the finite additivity of $V_{\varphi}$ is clear. Hence, it suffices to show that $V_{\varphi}$ is countably additive in the total variation norm sense. Suppose $\varphi$ is a positive measure on $(\mathbb{R}, \mathcal{B}(\mathbb{R}))$. In this case, $d P_{\varphi}=d P_{|\varphi|}$. Let $\left\langle B_{n}\right\rangle$ be a sequence of disjoint sets in $\mathcal{B}(C[0, t])$ with $B=\bigcup_{j=1}^{\infty} B_{j}$. Then for $E$ in $\mathcal{B}(\mathbb{R})$,

$$
\begin{aligned}
\int_{E} \widetilde{\psi}_{B, \varphi}(\xi) d P_{\varphi}(\xi) & \\
& \stackrel{(1)}{=} \int_{J_{t}^{-1}(E)} \chi_{B}(x) d \omega_{\varphi}(x) \\
& \stackrel{(2)}{=} \sum_{j=1}^{\infty} \int_{J_{t}^{-1}(E)} \chi_{B_{j}}(x) d \omega_{\varphi}(x) \\
& \stackrel{(3)}{=} \sum_{j=1}^{\infty} \int_{E} \widetilde{\psi}_{B_{j}, \varphi}(\xi) d P_{\varphi}(\xi) \\
& \stackrel{(4)}{=} \int_{E} \sum_{j=1}^{\infty} \widetilde{\psi}_{B_{j}, \varphi}(\xi) d P_{\varphi}(\xi) .
\end{aligned}
$$

Steps (1) and (3) follow from equality (4.10). Steps (2) and (4) result from the monotone convergence theorem.

Since $E$ is an arbitrary Borel set in (4.13), by [22, Theorem 8.1, p. 76],

$$
\widetilde{\psi}_{B, \varphi}(\xi)=\sum_{j=1}^{\infty} \widetilde{\psi}_{B_{j}, \varphi}(\xi)
$$

for $P_{\varphi}$-a.e. $\xi$. 
Since $\widetilde{\psi}_{B_{j}, \varphi} \geq 0$ for all natural numbers $j$, by [16], $\sum_{j=1}^{\infty} \widetilde{\psi}_{B_{j}, \varphi}$ converges to $\widetilde{\psi}_{B, \varphi}$ in the $L^{1}\left(\mathbb{R}, P_{\varphi}\right)$-norm sense. Hence

$$
\begin{aligned}
& \left|\sum_{j=k}^{\infty} V_{\varphi}\left(B_{j}\right)\right|(\mathbb{R}) \\
& \quad=\sum_{j=k}^{\infty} \int_{\mathbb{R}}\left[\int_{\mathbb{R}} \frac{1}{\sqrt{2 \pi t}} \widetilde{\psi}_{B_{j}, \varphi}(\xi) \exp \left\{-\frac{(\xi-u)^{2}}{2 t}\right\} d m_{L}(\xi)\right] d \varphi(u) \\
& \quad=\sum_{j=k}^{\infty}\left\|\widetilde{\psi}_{B_{j}, \varphi}\right\|_{L^{1}\left(\mathbb{R}, P_{\varphi}\right)} \\
& \quad \rightarrow 0
\end{aligned}
$$

as $k \rightarrow+\infty$.

Hence, $V_{\varphi}$ is a measure-valued measure in the total variation norm sense if $\varphi$ is a positive measure.

Now, let $\varphi$ be in $\mathcal{M}(\mathbb{R})$ with the Jordan decomposition $\varphi=\sum_{j=1}^{4} \alpha_{j} \varphi_{j}$ and let $\left\langle B_{j}\right\rangle$ be a sequence of disjoint sets in $\mathcal{B}(C[0, t])$ with $B=\bigcup_{j=1}^{\infty} B_{j}$. Then

$$
\begin{aligned}
\mid \sum_{j=k}^{\infty} & V_{\varphi}\left(B_{j}\right) \mid(\mathbb{R}) \\
& \leq \sum_{j=k}^{\infty}\left[\left(\sum_{n=1}^{2}\left\|\widetilde{\psi}_{B_{j}, \varphi_{n}}\right\|_{L^{1}\left(\mathbb{R}, P_{\varphi}\right)}\right)^{2}+\left(\sum_{n=1}^{2}\left\|\widetilde{\psi}_{B_{j}, \varphi_{n+2}}\right\|_{L^{1}\left(\mathbb{R}, P_{\varphi}\right)}\right)^{2}\right]^{\frac{1}{2}} \\
& \leq \sum_{j=k}^{\infty} \sum_{n=1}^{4}\left\|\widetilde{\psi}_{B_{j}, \varphi_{n}}\right\|_{L^{1}\left(\mathbb{R}, P_{\varphi}\right)} \\
& \rightarrow 0
\end{aligned}
$$

as $k \rightarrow+\infty$, as desired.

Theorem 4.3. Let $\varphi$ be in $\mathcal{M}(\mathbb{R})$ and let $B$ be in $\mathcal{B}(C[0, t])$ with $\left|\omega_{\varphi}\right|(B)=0$. Then $V_{\varphi}(B)$ is a zero measure on $(\mathbb{R}, \mathcal{B}(\mathbb{R}))$.

Proof. Let $\varphi=\sum_{j=1}^{4} \alpha_{j} \varphi_{j}$ be the Jordan decomposition. Then $\omega_{\varphi_{j}}(B)=0$ for $j=1,2,3,4$; so for $j=1,2,3,4, \widetilde{\psi}_{B, \varphi_{j}}=0 \quad P_{\varphi_{j}}$-a.e., which implies that for $j=1,2,3,4, \widetilde{\psi}_{B, \varphi_{j}}=0 m_{L}$-a.e. Hence $\psi_{B, \varphi}=0, m_{L}$-a.e. By (4.10) and (4.11), for $E$ in $\mathcal{B}(\mathbb{R}),\left[V_{\varphi}(B)\right](E)=0$, as desired.

Theorem 4.4. Let $\varphi$ be in $\mathcal{M}(\mathbb{R})$ and let $\vec{t}=\left(t_{0}, t_{1}, \cdots, t_{n}\right)$ be a vector in $\mathbb{R}^{n+1}$ with $0=t_{0}<\cdots<t_{n}=t$. Let $f: \mathbb{R}^{n+1} \rightarrow \mathbb{C}$ be a Borel measurable function such that $f\left(u_{0}, u_{1}, \cdots, u_{n}\right) W\left(n+1 ; \vec{t} ; u_{0}, \cdots, u_{n}\right)$ is $|\varphi| \times \prod_{j=1}^{n} m_{L}$-integrable. Let $F: C[0, t] \rightarrow \mathbb{C}$ be a function with $F(x)=\left(f \circ J_{\vec{t}}\right)(x)=f\left(x\left(t_{0}\right), x\left(t_{1}\right), \cdots, x\left(t_{n}\right)\right)$. 
Then $F$ is $V_{\varphi}$-Bartle integrable on $C[0, t]$ and for $E$ in $\mathcal{B}(\mathbb{R})$,

$$
\begin{aligned}
& {\left[(B a)-\int_{C[0, t]} F(x) d V_{\varphi}(x)\right](E)} \\
& \quad=\int_{E}\left\{\int _ { \mathbb { R } ^ { n - 1 } } \left(\int_{\mathbb{R}} f\left(u_{0}, u_{1}, \cdots, u_{n}\right) W\left(n+1 ; \vec{t} ; u_{0}, \cdots, u_{n}\right)\right.\right. \\
& \left.\left.d \varphi\left(u_{0}\right)\right) d\left(\prod_{j=1}^{n-1} m_{L}\right)\left(u_{1}, \cdots, u_{n-1}\right)\right\} d m_{L}\left(u_{n}\right) .
\end{aligned}
$$

Proof. Let $f=\chi_{B}$ where $B$ is a Borel subset of $\mathbb{R}^{n+1}$. Then $F(x)=\left(\chi_{B} \circ J_{\vec{t}}\right)(x)$ $=\chi_{J_{\vec{t}}^{-1}(B)}(x)$ is $V_{\varphi}$-Bartle integrable and for $E$ in $\mathcal{B}(\mathbb{R})$,

$$
\begin{aligned}
{[(B a)-} & \left.\int_{C[0, t]} F(x) d V_{\varphi}(x)\right](E) \\
= & {\left[V_{\varphi}\left(J_{\vec{t}}^{-1}(B)\right)\right](E) } \\
= & \omega_{\varphi}\left(J_{\vec{t}}^{-1}(B) \cap J_{t}^{-1}(E)\right) \\
= & \int_{E}\left\{\int _ { \mathbb { R } ^ { n - 1 } } \left(\int_{\mathbb{R}} \chi_{B}\left(u_{0}, u_{1}, \cdots, u_{n}\right) W\left(n+1 ; \vec{t} ; u_{0}, \cdots, u_{n}\right)\right.\right. \\
& \left.\left.d \varphi\left(u_{0}\right)\right) d\left(\prod_{j=1}^{n-1} m_{L}\right)\left(u_{1}, u_{2}, \cdots, u_{n-1}\right)\right\} d m_{L}\left(u_{n}\right) .
\end{aligned}
$$

If $f$ is a simple function, then by the basic properties of the Lebesgue integral and the Bartle integral, it is not hard to show that $F$ is $V_{\varphi}$-Bartle integrable and the equality (4.16) holds for $f$. We assume that $\varphi$ is a positive measure in $\mathcal{M}(\mathbb{R})$ and $f$ is a nonnegative real-valued Borel measurable function on $\mathbb{R}^{n+1}$ such that $f\left(u_{0}, u_{1}, \cdots, u_{n}\right) W\left(n+1 ; \vec{t} ; u_{0}, \cdots, u_{n}\right)$ is $|\varphi| \times \prod_{j=1}^{n} m_{L}$-integrable. Then there is a sequence $\left\langle f_{m}\right\rangle$ of nonnegative real-valued simple functions such that $\left\langle f_{m}\left(u_{0}, u_{1}, \cdots, u_{n}\right)\right\rangle$ is increasing for $\left(u_{0}, u_{1}, \cdots, u_{n}\right)$ in $\mathbb{R}^{n+1},\left\langle f_{m}\right\rangle$ converges to $f$ for $|\varphi| \times \prod_{j=1}^{n} m_{L}$-a.e. and

$$
\left\langle\int_{\mathbb{R}^{n+1}} f_{m}\left(u_{0}, u_{1}, \cdots, u_{n}\right) W\left(n+1 ; \vec{t} ; u_{0}, \cdots, u_{n}\right) d\left(\varphi \times \prod_{j=1}^{n} m_{L}\right)\left(u_{0},\left(u_{1}, \cdots, u_{n}\right)\right)\right\rangle
$$

converges to

$$
\int_{\mathbb{R}^{n+1}} f\left(u_{0}, u_{1}, \cdots, u_{n}\right) W\left(n+1 ; \vec{t} ; u_{0}, \cdots, u_{n}\right) d\left(\varphi \times \prod_{j=1}^{n} m_{L}\right)\left(u_{0},\left(u_{1}, \cdots, u_{n}\right)\right)
$$


Let $N=\left\{\left(u_{0}, u_{1}, \cdots, u_{n}\right)\right.$ in $\mathbb{R}^{n+1} \mid$ either the limit $\lim _{m \rightarrow \infty} f_{m}\left(u_{0}, u_{1}, \cdots, u_{n}\right)$ does not exist or the limit $\lim _{m \rightarrow \infty} f_{m}\left(u_{0}, u_{1}, \cdots, u_{n}\right)$ exists but is not equal to $f\left(u_{0}, u_{1}\right.$, $\left.\left.\cdots, u_{n}\right)\right\}$. Then $N$ is $|\varphi| \times \prod_{j=1}^{n} m_{L}$-null. By Theorem 3.1, $J_{\vec{t}}^{-1}(N)$ is $\omega_{|\varphi|}$-null. For a natural number $m$ and for $E$ in $\mathcal{B}(\mathbb{R})$, let

$$
F_{m}=f_{m} \circ J_{\vec{t}}
$$

and

$$
\begin{aligned}
\mu_{m}(E)= & \int_{E}\left\{\int_{\mathbb{R}^{n-1}}\left(\int_{\mathbb{R}} f_{m}\left(u_{0}, u_{1}, \cdots, u_{n}\right) W\left(n+1 ; \vec{t} ; u_{0}, \cdots, u_{n}\right) d \varphi\left(u_{0}\right)\right)\right. \\
& \left.d\left(\prod_{j=1}^{n-1} m_{L}\right)\left(u_{1}, \cdots, u_{n-1}\right)\right\} d m_{L}\left(u_{n}\right) .
\end{aligned}
$$

Then $\left\langle F_{m}\right\rangle$ converges to $F \omega_{|\varphi|}$-a.e.; so by Theorem 4.3, $\left\langle F_{m}\right\rangle$ converges to $F\left\|V_{\varphi}\right\|$ a.e. By the monotone convergence theorem, for $E$ in $\mathcal{B}(\mathbb{R}),\left\langle\mu_{m}(E)\right\rangle$ converges; here, we denote $\mu(E)=\lim _{m \rightarrow \infty} \mu_{m}(E)$. By the Vitali-Hahn-Saks theorem, $\mu$ is a countably additive measure on $\mathcal{B}(\mathbb{R})$ and for $E$ in $\mathcal{B}(\mathbb{R})$,

$$
\begin{aligned}
\mu(E) & =\int_{E}\left\{\int_{\mathbb{R}^{n-1}}\left(\int_{\mathbb{R}} f\left(u_{0}, u_{1}, \cdots, u_{n}\right) W\left(n+1 ; \vec{t} ; u_{0}, u_{1}, \cdots, u_{n}\right) d \varphi\left(u_{0}\right)\right)\right. \\
& \left.d\left(\prod_{j=1}^{n-1} m_{L}\right)\left(u_{1}, \cdots, u_{n-1}\right)\right\} d m_{L}\left(u_{n}\right) .
\end{aligned}
$$

Since for a natural number $m,\left|f_{m}-f\right| \leq 2|f|$, by the dominated convergence theorem,

$$
\begin{aligned}
& \overline{\lim _{m \rightarrow \infty}}\left|\mu_{m}-\mu\right|(\mathbb{R}) \\
& \leq \lim _{m \rightarrow \infty} \int_{\mathbb{R}^{n+1}}\left|f_{m}\left(u_{0}, u_{1}, \cdots, u_{n}\right)-f\left(u_{0}, u_{1}, \cdots, u_{n}\right)\right| \\
& \\
& W\left(n+1 ; \vec{t} ; u_{0}, u_{1}, \cdots, u_{n}\right) d\left(|\varphi| \times \prod_{j=1}^{n} m_{L}\right)\left(u_{0}, u_{1}, \cdots, u_{n}\right) \\
& =0 .
\end{aligned}
$$


Hence $\left\langle\mu_{m}\right\rangle$ converges to $\mu$ in the total variation norm sense; so $F$ is $V_{\varphi}$-Bartle integrable. Moreover, for $E$ in $\mathcal{B}(\mathbb{R})$,

$$
\begin{aligned}
{[(B a)-} & \left.\int_{C[0, t]} F(x) d V_{\varphi}(x)\right](E) \\
= & \lim _{m \rightarrow \infty}\left[(B a)-\int_{C[0, t]} F_{m}(x) d V_{\varphi}(x)\right](E) \\
= & \lim _{m \rightarrow \infty} \int_{E}\left\{\int _ { \mathbb { R } ^ { n - 1 } } \left(\int_{\mathbb{R}} f_{m}\left(u_{0}, u_{1}, \cdots, u_{n}\right) W\left(n+1 ; \overrightarrow{t ;} u_{0}, \cdots, u_{n}\right)\right.\right. \\
& \left.\left.d \varphi\left(u_{0}\right)\right) d\left(\prod_{j=1}^{n-1} m_{L}\right)\left(u_{1}, u_{2}, \cdots, u_{n-1}\right)\right\} d m_{L}\left(u_{n}\right) \\
= & \int_{E}\left\{\int _ { \mathbb { R } ^ { n - 1 } } \left(\int_{\mathbb{R}} f\left(u_{0}, u_{1}, \cdots, u_{n}\right) W\left(n+1 ; \vec{t}^{\prime} u_{0}, \cdots, u_{n}\right)\right.\right. \\
& \left.\left.d \varphi\left(u_{0}\right)\right) d\left(\prod_{j=1}^{n-1} m_{L}\right)\left(u_{1}, u_{2}, \cdots, u_{n-1}\right)\right\} d m_{L}\left(u_{n}\right) \cdot
\end{aligned}
$$

Now, it remains to show that this theorem holds in the general case. Let $\varphi$ in $\mathcal{M}(\mathbb{R})$ with the Jordan decomposition $\varphi=\sum_{j=1}^{4} \alpha_{j} \varphi_{j}$ and let $f: \mathbb{R}^{n+1} \rightarrow \mathbb{C}$ be a Borel measurable function such that $f\left(u_{0}, u_{1}, \cdots, u_{n}\right) W\left(n+1 ; \vec{t} ; u_{0}, u_{1}, \cdots, u_{n}\right)$ is $|\varphi| \times \prod_{j=1}^{n} m_{L}$-integrable. Then we can write $f=\sum_{j=1}^{4} \alpha_{j} f_{j}$ where $f_{j}$ is a nonnegative Borel measurable function for $j=1,2,3,4$. Then $f_{j}\left(u_{0}, u_{1}, \cdots, u_{n}\right) W(n+$ $\left.1 ; \vec{t} ; u_{0}, u_{1}, \cdots, u_{n}\right)$ is $\varphi_{k} \times \prod_{j=1}^{n} m_{L}$-integrable for $j, k=1,2,3,4$, and we proved the following fact already. Letting $F_{j}=f_{j} \circ J_{\vec{t}}$ for $j=1,2,3,4, F_{j}$ is $V_{\varphi_{k}}$-Bartle integrable for $j, k=1,2,3,4$; and for $j, k=1,2,3,4$ and for $E$ in $\mathcal{B}(\mathbb{R})$, we then have

$$
\begin{aligned}
& {\left[(B a)-\int_{C[0, t]} F_{j}(x) d V_{\varphi_{k}}(x)\right](E)} \\
& \quad=\int_{E}\left\{\int _ { \mathbb { R } ^ { n - 1 } } \left(\int_{\mathbb{R}^{\prime}} f_{j}\left(u_{0}, u_{1}, \cdots, u_{n}\right) W\left(n+1 ; \vec{t} ; u_{0}, u_{1}, \cdots, u_{n}\right)\right.\right. \\
& \left.\left.d \varphi_{k}\left(u_{0}\right)\right) d\left(\prod_{p=1}^{n-1} m_{L}\right)\left(u_{1}, u_{2}, \cdots, u_{n-1}\right)\right\} d m_{L}\left(u_{n}\right) .
\end{aligned}
$$


By (3) in Remark 4.1 and the basic properties of the Lebesgue integral and the Bartle integral, for $E$ in $\mathcal{B}(\mathbb{R})$,

$$
\begin{aligned}
{[(B a)} & \left.-\int_{C[0, t]} F(x) d V_{\varphi}(x)\right](E) \\
= & \sum_{j, k=1}^{4} \alpha_{j} \alpha_{k}\left[(B a)-\int_{C[0, t]} F_{j}(x) d V_{\varphi_{k}}(x)\right](E) \\
= & \sum_{j, k=1}^{4} \alpha_{j} \alpha_{k} \int_{E}\left\{\int _ { \mathbb { R } ^ { n - 1 } } \left(\int_{\mathbb{R}} f_{j}\left(u_{0}, u_{1}, \cdots, u_{n}\right) W\left(n+1 ; \vec{t} ; u_{0}, \cdots, u_{n}\right)\right.\right. \\
& \left.\left.d \varphi_{k}\left(u_{0}\right)\right) d\left(\prod_{p=1}^{n-1} m_{L}\right)\left(u_{1}, \cdots, u_{n-1}\right)\right\} d m_{L}\left(u_{n}\right) \\
= & \int_{E}\left\{\int _ { \mathbb { R } ^ { n - 1 } } \left(\int_{\mathbb{R}}^{n-1} f\left(u_{0}, u_{1}, \cdots, u_{n}\right) W\left(n+1 ; \vec{t} ; u_{0}, \cdots, u_{n}\right)\right.\right. \\
& \left.\left.d \varphi\left(u_{0}\right)\right) d\left(\prod_{p=1}^{n-1} m_{L}\right)\left(u_{1}, \cdots, u_{n-1}\right)\right\} d m_{L}\left(u_{n}\right),
\end{aligned}
$$

as desired.

\section{A measure-Valued Feynman-Kac formula}

In this section, we will achieve the measure-valued Feynman-Kac formula for the integral with respect to a measure-valued measure $V_{\varphi}$ of a suitable functional.

Theorem 5.1. Let $\varphi$ be in $\mathcal{M}(\mathbb{R})$, let $\eta$ be a complex-valued Borel measure on $[0, t]$ and let $\theta$ be in $L_{\varphi ; \infty, 1 ; \eta}$. Then

$$
|\theta(s, x(s))| \leq\|\theta(s, \cdot)\|_{\varphi ; \infty}
$$

for $|\eta| \times \omega_{|\varphi|}$-a.e. $(s, x)$ in $[0, t] \times C[0, t]$.

Proof. We consider a propositional function $P:[0, t] \times \mathbb{R} \rightarrow\{0,1\}$ given by $P(s, v)=1$ if either $\theta(0, v)$ fails to be defined $|\varphi|$-a.e. and $\theta(s, v)$ fails to be defined $m_{L}$-a.e. for $0<s \leq t$ or $\theta(s, v)$ is defined but $|\theta(s, v)|>\|\theta(s, \cdot)\|_{\varphi ; \infty}$ and $P(s, v)=0$ otherwise. Let $N=\{(s, v)$ in $[0, t] \times \mathbb{R} \mid P(s, v)=1\}, N_{1}=$ $\{(0, v)$ in $[0, t] \times \mathbb{R} \mid P(0, v)=1\}$ and $N_{2}=\{(s, v)$ in $[0, t] \times \mathbb{R} \mid P(s, v)=1$ and $0<$ $s \leq t\}$. Then both $N_{1}$ and $N_{2}$ are disjoint Borel subsets and $N=N_{1} \cup N_{2}$. Let $H:[0, t] \times C[0, t] \rightarrow[0, t] \times \mathbb{R}$ be a function with $H(s, x)=(s, x(s))$. Then $H$ is continuous and $\theta(s, x(s))=(\theta \circ H)(s, x)$ is Borel measurable with respect to $(s, x)$. Since $\left[H^{-1}(N)\right]^{(0)}=\left[H^{-1}\left(N_{1}\right)\right]^{(0)}$ and $\left[H^{-1}(N)\right]^{(s)}=\left[H^{-1}\left(N_{2}\right)\right]^{(s)}$ for $0<s \leq t$, by Theorem 3.1, $\omega_{|\varphi|}\left(\left[H^{-1}(N)\right]^{(0)}\right)=|\varphi|\left(\left[N_{1}\right]^{(0)}\right)=0$ and $\omega_{|\varphi|}\left(\left[H^{-1}(N)\right]^{(s)}\right)=$ $\int_{\left[N_{2}\right]^{(s)}} \frac{1}{\sqrt{2 \pi s}}\left(\int_{\mathbb{R}} \exp \left\{-\frac{\left(u-u_{0}\right)^{2}}{2 s}\right\} d|\varphi|\left(u_{0}\right)\right) d m_{L}(u)=0$ for $0<s \leq t$; that is, $\omega_{|\varphi|}\left(\left[H^{-1}(N)\right]^{(s)}\right)=0$ for all $s$ in $[0, t]$ where $[A]^{(s)}$ is the $s$-cross section of $A$. 
By the Fubini theorem,

$$
\begin{aligned}
|\eta| \times & \omega_{|\varphi|}\left(H^{-1}(N)\right) \\
& =\int_{[0, t] \times C[0, t]} \chi_{H^{-1}(N)}(s, x) d\left(|\eta| \times \omega_{|\varphi|}\right)(s, x) \\
& =\int_{[0, t]} \omega_{|\varphi|}\left(\left[H^{-1}(N)\right]^{(s)}\right) d|\eta|(s) \\
& =0
\end{aligned}
$$

as desired.

Throughout this section, let $\eta=\mu+\nu$ be a complex-valued Borel measure on $[0, t]$ such that $\mu$ is the continuous part of $\eta$ and $\nu=\sum_{p=0}^{n} c_{p} \delta_{\tau_{p}}$ where $0=\tau_{0}<$ $\tau_{1}<\tau_{2}<\cdots<\tau_{n}=t$ and $c_{p}(p=0,1, \cdots, n)$ are complex numbers, let $\varphi$ be in $\mathcal{M}(\mathbb{R})$ and let $\theta$ be in $L_{\varphi ; \infty, 1 ; \eta}$. For nonnegative integers $q$ and $j_{1}, \cdots, j_{n}$ with $q=j_{1}+j_{2}+\cdots+j_{n}$, let

$$
\begin{aligned}
\triangle_{q ; j_{1}, j_{2}, \cdots, j_{n}} & \\
= & \left\{\left(s_{1,1}, s_{1,2}, \cdots, s_{1, j_{1}}, s_{2,1}, \cdots, s_{n-1, j_{n-1}}, s_{n, 1}, \cdots, s_{n, j_{n}}\right) \mid \tau_{0}=0<s_{1,1}<\right. \\
& \left.\cdots<s_{1, j_{1}}<\tau_{1}<s_{2,1}<\cdots<\tau_{n-1}<s_{n, 1}<\cdots<s_{n, j_{n}}<\tau_{n}=t\right\} .
\end{aligned}
$$

For convenience, we let $M_{\theta(s, \cdot)} \equiv M_{\theta(s)}$ for $0 \leq s \leq t$ and $\tau_{0}=s_{0,0}, \tau_{n}=t=$ $s_{n, j_{n}+1}$ and $\tau_{k}=s_{k+1,0}=s_{k, j_{k}+1}$ for $k=1,2, \cdots, n-1$. For nonnegative integers $m, q_{0}, \cdots, q_{n+1}, j_{1}, \cdots, j_{n}$ with $m=q_{0}+q_{1}+\cdots+q_{n+1}$ and $q_{n+1}=j_{1}+j_{2}+\cdots+j_{n}$, let $K(m, n, q, j): \triangle_{q_{n+1} ; j_{1}, j_{2}, \cdots, j_{n}} \times C[0, t] \rightarrow \mathbb{C}$ be a function with

$$
\begin{aligned}
K(m, n, q, j)\left(\left(s_{1,1}, \cdots, s_{n, j_{n}}\right), x\right) \\
\quad=\left[\prod_{i=0}^{n} \theta\left(\tau_{i}, x\left(\tau_{i}\right)\right)^{q_{i}}\right]\left[\prod_{i=1}^{n} \prod_{j=1}^{j_{i}} \theta\left(s_{i, j}, x\left(s_{i, j}\right)\right)\right]
\end{aligned}
$$

and let $D(m, n, q, j): \triangle_{q_{n+1} ; j_{1}, j_{2}, \cdots, j_{n}} \rightarrow \mathbb{R}$ be a function with

$$
\begin{aligned}
& D(m, n, q, j)\left(s_{1,1}, \cdots, s_{n, j_{n}}\right) \\
&=\left[\prod_{i=0}^{n}\left\|\theta\left(\tau_{i}, \cdot\right)\right\|_{\varphi ; \infty}^{q_{i}}\right]\left[\prod_{i=1}^{n} \prod_{j=1}^{j_{i}}\left\|\theta\left(s_{i, j}, \cdot\right)\right\|_{\varphi ; \infty}\right] .
\end{aligned}
$$

Lemma 5.2. (1) $|K(m, n, q, j)| \leq D(m, n, q, j) \quad|\mu| \times \omega_{|\varphi|}$-a.e.,

(2) $\left|\int_{\triangle_{q_{n+1} ; j_{1}, \cdots, j_{n}}} D(m, n, q, j)\left(s_{1,1}, \cdots, s_{n, j_{n}}\right) d\left(\prod_{i=1}^{n} \prod_{j=1}^{j_{i}} \mu\right)\left(s_{1,1}, \cdots, s_{n, j_{n}}\right)\right|$ $\leq \frac{1}{q_{n+1} !}\left(\prod_{i=0}^{n}\left\|\theta\left(\tau_{i}, \cdot\right)\right\|_{\varphi ; \infty}^{q_{i}}\right)\left(\|\theta\|_{\varphi ; \infty, 1 ; \mu}\right)^{q_{n+1}}$ and

(3) $D(m, n, q, j)$ is $\left(\prod_{i=1}^{n} \prod_{j=1}^{j_{i}} \mu\right) \times V_{\varphi}$-Bartle integrable on $\triangle_{q_{n+1} ; j_{1}, \cdots, j_{n}} \times$ $C[0, t]$.

Proof. By Theorem 5.1, the statement (1) in this lemma is clear. Obviously, $D(m, n, q, j)$ is Borel measurable. Letting

$$
\triangle_{q_{n+1}}=\left\{\left(s_{1}, s_{2}, \cdots, s_{q_{n+1}}\right) \text { in }[0, t]^{q_{n+1}} \mid 0=s_{0}<s_{1}<s_{2}<\cdots<s_{q_{n+1}}<t\right\},
$$


since $\triangle_{q_{n+1}}$ is Borel measurable and $\triangle_{q_{n+1} ; j_{1}, \cdots, j_{n}} \subset \triangle_{q_{n}}$,

$$
\begin{aligned}
& \left|\int_{\triangle_{q_{n}+1 ; j_{1}, \cdots, j_{n}}} D(m, n, q, j)\left(s_{1,1}, \cdots, s_{n, j_{n}}\right) d\left(\prod_{i=1}^{n} \prod_{j=1}^{j_{i}} \mu\right)\left(s_{1,1}, \cdots, s_{n, j_{n}}\right)\right| \\
& \quad \leq\left(\prod_{i=0}^{n}\left\|\theta\left(\tau_{i}, \cdot\right)\right\|_{\varphi ; \infty}^{q_{i}}\right) \int_{\triangle_{q_{n+1}}} \prod_{i=1}^{q_{n+1}}\left\|\theta\left(s_{i}, \cdot\right)\right\|_{\varphi ; \infty} d\left(\prod_{i=1}^{q_{n+1}}|\mu|\right)\left(s_{1}, s_{2}, \cdots, s_{q_{n+1}}\right) \\
& \quad=\frac{1}{q_{n+1} !}\left[\int_{[0, t]}\|\theta(s, \cdot)\|_{\varphi ; \infty} d|\mu|(s)\right]^{q_{n+1}}\left[\prod_{i=0}^{n}\left\|\theta\left(\tau_{i}, \cdot\right)\right\|_{\varphi ; \infty}^{q_{i}}\right] \\
& =\frac{1}{q_{n+1} !}\left(\|\theta\|_{\varphi ; \infty, 1 ; \mu}\right)^{q_{n+1}}\left(\prod_{i=0}^{n}\left\|\theta\left(\tau_{i}, \cdot\right)\right\|_{\varphi ; \infty}^{q_{i}}\right) .
\end{aligned}
$$

Hence, we proved statement (2) in this lemma. Since $D(m, n, q, j)$ is $\prod_{i=1}^{n} \prod_{j=1}^{j_{i}}|\mu|$ integrable, there is a sequence $\left\langle g_{k}\right\rangle$ of simple functions such that $\left\langle g_{k}\right\rangle$ converges to $D(m, n, q, j) \quad \prod_{i=1}^{n} \prod_{j=1}^{j_{i}}|\mu|$-a.e. and a sequence $\left\langle\int_{\triangle_{q_{n+1} ; j_{1}, \cdots, j_{n}}} g_{k}\left(s_{1,1}, \cdots, s_{n, j_{n}}\right)\right.$ $\left.d\left(\prod_{i=1}^{n} \prod_{j=1}^{j_{i}} \mu\right)\left(s_{1,1}, \cdots, s_{n, j_{n}}\right)\right\rangle$ is Cauchy. Then $\left\langle g_{k}\right\rangle$ converges to $D(m, n, q, j)$ $\prod_{i=1}^{n} \prod_{j=1}^{j_{i}}|\mu| \times\left\|V_{\varphi}\right\|$-a.e. and for two natural numbers $k$ and $l$,

$$
\begin{gathered}
\mid(B a)-\int_{\triangle_{q_{n+1} ; j_{1}, \cdots, j_{n}}}\left(g_{l}-g_{k}\right)\left(s_{1,1}, \cdots, s_{n, j_{n}}\right) d\left[\left(\prod_{i=1}^{n} \prod_{j=1}^{j_{i}} \mu\right) \times V_{\varphi}\right] \\
\left(\left(s_{1,1}, \cdots, s_{n, j_{n}}\right), x\right) \mid \\
\leq 4|\varphi|(\mathbb{R}) \mid \int_{\triangle_{q_{n+1} ; j_{1}, \cdots, j_{n}}}\left(g_{l}-g_{k}\right)\left(s_{1,1}, \cdots, s_{n, j_{n}}\right) \\
d\left(\prod_{i=1}^{n} \prod_{j=1}^{j_{i}} \mu\right)\left(s_{1,1}, \cdots, s_{n, j_{n}}\right) \mid .
\end{gathered}
$$

So

$$
\left\langle(B a)-\int_{\triangle_{q_{n+1} ; j_{1}, \cdots, j_{n}}} g_{k}\left(s_{1,1}, \cdots, s_{n, j_{n}}\right) d\left[\left(\prod_{i=1}^{n} \prod_{j=1}^{j_{i}} \mu\right) \times V_{\varphi}\right]\left(\left(s_{1,1}, \cdots, s_{n, j_{n}}\right), x\right)\right\rangle
$$

is Cauchy. Therefore, $D(m, n, q, j)$ is $\left(\prod_{i=1}^{n} \prod_{j=1}^{j_{i}} \mu\right) \times V_{\varphi}$-Bartle integrable.

Lemma 5.3. $\theta(s, x(s))$ is $\mu \times V_{\varphi}$-Bartle integrable on $[0, t] \times C[0, t]$.

Proof. Since $\theta$ is Borel measurable on $[0, t] \times \mathbb{R}$, there is a sequence $\left\langle\psi_{n}\right\rangle$ of simple functions on $[0, t] \times \mathbb{R}$ such that $\left\langle\psi_{n}\right\rangle$ converges to $\theta \quad|\mu| \times m_{L}$-a.e. Let $N=$ $\left\{(s, u)\right.$ in $[0, t] \times \mathbb{R} \mid$ either $\theta(s, u)$ is not defined or $\left\langle\psi_{n}(s, u)\right\rangle$ does not converge to $\theta(s, u)\}$ and let $H:[0, t] \times C[0, t] \rightarrow[0, t] \times \mathbb{R}$ be a function with $H(s, x)=(s, x(s))$. Then $H$ is continuous, and so the sequence $\left\langle\psi_{n} \circ H\right\rangle$ is a sequence of simple functions such that $\left\langle\psi_{n} \circ H\right\rangle$ converges to $\theta \circ H$ on $[0, t] \times C[0, t] \backslash H^{-1}(N)$. Moreover, by 
WIENER MEASURE AND THE MEASURE-VALUED FEYNMAN-KAC FORMULA 4941

the Fubini theorem and Theorem 4.4,

$$
\begin{aligned}
|\mu| \times & \omega_{|\varphi|}\left(H^{-1}(N)\right) \\
& =\int_{[0, t]} \int_{C[0, t]} \chi_{H^{-1}(N)}(s, x) d \omega_{|\varphi|}(x) d|\mu|(s) \\
& =\int_{[0, t]} \int_{C[0, t]} \chi_{[N]^{(s)}}(x(s)) d \omega_{|\varphi|}(x) d|\mu|(s) \\
& =\int_{(0, t]} \int_{C[0, t]} \chi_{[N]^{(s)}}(x(s)) d \omega_{|\varphi|}(x) d|\mu|(s) \\
& =\int_{(0, t]} \frac{1}{\sqrt{2 \pi s}} \int_{\mathbb{R}} \chi_{[N]^{(s)}}\left(u_{1}\right) \exp \left\{-\frac{\left(u_{1}-u_{0}\right)^{2}}{2 s}\right\} d m_{L}\left(u_{1}\right) d \varphi\left(u_{0}\right) d|\mu|(s) \\
& =0 ;
\end{aligned}
$$

so $|\mu| \times \omega_{|\varphi|}\left(H^{-1}(N)\right)=0$. If $|\mu| \times \omega_{|\varphi|}\left(H^{-1}(N)\right)=0$, then $|\mu|$-a.e. $s,\left[H^{-1}(N)\right]^{(s)}$

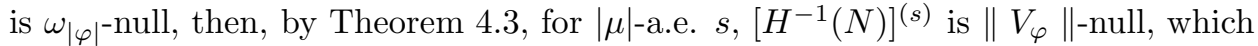
implies that for $x^{*}$ in $\mathcal{R} \mathcal{M}^{*}(\mathbb{R})$ with $\left\|x^{*}\right\|_{\infty} \leq 1,\left[H^{-1}(N)\right]^{(s)}$ is $\left|x^{*} V_{\varphi}\right|$-null for $|\mu|$-a.e. $s$. Thus, we have

$$
\begin{aligned}
\| \mu & \times V_{\varphi} \|\left(H^{-1}(N)\right) \\
& =\sup \left\{\left|x^{*}\left(\mu \times V_{\varphi}\right)\right|\left(H^{-1}(N)\right) \mid x^{*} \text { is in } L^{\infty}\left(\mathbb{R}, m_{L}\right) \text { and }\left\|x^{*}\right\|_{\infty} \leq 1\right\} \\
& =\sup \left\{\left|\mu \times\left(x^{*} V_{\varphi}\right)\right|\left(H^{-1}(N)\right) \mid x^{*} \text { is in } L^{\infty}\left(\mathbb{R}, m_{L}\right) \text { and }\left\|x^{*}\right\|_{\infty} \leq 1\right\} \\
& =\sup \left\{|\mu| \times\left|x^{*} V_{\varphi}\right|\left(H^{-1}(N)\right) \mid x^{*} \text { is in } L^{\infty}\left(\mathbb{R}, m_{L}\right) \text { and }\left\|x^{*}\right\|_{\infty} \leq 1\right\} \\
& =0 .
\end{aligned}
$$

So, the sequence $\left\langle\psi_{n} \circ H\right\rangle$ of simple functions on $[0, t] \times C[0, t]$ converges to $\theta \circ H$ $\left\|\mu \times V_{\varphi}\right\|$-a.e., which implies that $\theta(s, x(s))$ is $\mu \times V_{\varphi}$-measurable. Now, by (4.12),

$$
\begin{aligned}
\int_{[0, t] \times C[0, t]}|\theta(s, x(s))| d\left|x^{*}\left(\mu \times V_{\varphi}\right)\right|(s, x) \\
\quad \leq \int_{[0, t] \times C[0, t]}|\theta(s, x(s))| d|\mu| \times\left\|V_{\varphi}\right\|(s, x) \\
\quad \leq \int_{C[0, t]} \int_{[0, t]}\|\theta(s, \cdot)\|_{\varphi ; \infty} d|\mu|(s) d\left\|V_{\varphi}\right\|(x) \\
\quad=\|\theta\|_{\varphi ; \infty, 1 ; \mu}\left\|V_{\varphi}\right\|(C[0, t]) \\
\quad \leq 4|\varphi|(\mathbb{R})\|\theta\|_{\varphi ; \infty, 1 ; \mu} \\
<+\infty,
\end{aligned}
$$

that is, $\theta(s, x(s))$ is $x^{*}\left(\mu \times V_{\varphi}\right)$-integrable. Now let $F: \mathcal{B}(\mathbb{R}) \rightarrow \mathbb{C}$ be a measure given by

$$
\begin{gathered}
F(E)=\int_{E} \int_{[0, t]} \int_{\mathbb{R}} \int_{\mathbb{R}} \theta\left(s, u_{1}\right) \frac{1}{\sqrt{(2 \pi)^{2} s(t-s)}} \exp \left\{-\frac{\left(u_{1}-u_{0}\right)^{2}}{2 s}-\frac{\left(u_{2}-u_{1}\right)^{2}}{2(t-s)}\right\} \\
d \varphi\left(u_{0}\right) d m_{L}\left(u_{1}\right) d \mu(s) d m_{L}\left(u_{2}\right),
\end{gathered}
$$

for $E$ in $\mathcal{B}(\mathbb{R})$. Then by the Tonelli theorem, we know that for $E$ in $\mathcal{B}(\mathbb{R}),|F(E)|$ $\leq\|\theta\|_{\varphi ; \infty, 1 ; \mu}$, that is, $F$ is well-defined. So $F$ is in $\mathcal{R M}(\mathbb{R})$. For $x^{*}$ in $\mathcal{R} \mathcal{M}^{*}(\mathbb{R})$, 
there exists a function $f$ in $L^{\infty}\left(\mathbb{R}, m_{L}\right)$ such that $x^{*}(\mu)=\int_{\mathbb{R}} f(\xi) d \mu(\xi)$,

$$
\begin{aligned}
x^{*}(F) & \stackrel{(1)}{=} \int_{\mathbb{R}} f\left(u_{2}\right) d F\left(u_{2}\right) \\
& \stackrel{(2)}{=} \int_{\mathbb{R}} f\left(u_{2}\right) \frac{d F}{d m_{L}}\left(u_{2}\right) d m_{L}\left(u_{2}\right) \\
& \stackrel{(3)}{=} \int_{\mathbb{R}} f\left(u_{1}\right)\left[\int _ { [ 0 , t ] } \int _ { \mathbb { R } } \int _ { \mathbb { R } } \theta ( s , u _ { 1 } ) \frac { 1 } { \sqrt { ( 2 \pi ) ^ { 2 } s ( t - s ) } } \operatorname { e x p } \left\{-\frac{\left(u_{1}-u_{0}\right)^{2}}{2 s}\right.\right. \\
& \left.-\frac{\left(u_{2}-u_{1}\right)^{2}}{2(t-s)} d \varphi\left(u_{0}\right) d m_{L}\left(u_{1}\right) d \mu(s)\right] d m_{L}\left(u_{2}\right) \\
& \int_{[0, t]}\left[\int _ { \mathbb { R } } f ( u _ { 1 } ) \left(\int _ { \mathbb { R } } \int _ { \mathbb { R } } \theta ( s , u _ { 1 } ) \frac { 1 } { \sqrt { ( 2 \pi ) ^ { 2 } s ( t - s ) } } \operatorname { e x p } \left\{-\frac{\left(u_{1}-u_{0}\right)^{2}}{2 s}\right.\right.\right. \\
& \stackrel{(5)}{=} \int_{[0, t]} \int_{C[0, t]} \theta(s, x(s)) d x^{*}\left(V_{\varphi} x\right) d \mu(s) \\
& \stackrel{(6)}{=} \int_{[0, t] \times C[0, t]} \theta(s, x(s)) d\left(\mu \times x_{1}\right)^{2} \\
& \left.\left.\stackrel{(7)}{=} \int_{[0, t] \times C[0, t]} \theta(s, x(s)) d x_{\varphi}^{*}\left(\mu \times u_{\varphi}\right) d m_{L}\left(u_{1}\right)\right) d m_{L}\left(u_{2}\right)\right] d \mu(s) \\
&
\end{aligned}
$$

Step (1) follows from (C) in section 2. By the Radon-Nikodym theorem, we have Step (2). From the definition of a measure $F$, we obtain Step (3). Steps (4) and (6) result from the Fubini theorem. By Theorem 4.4, $\theta(s, x(s))$ is $V_{\varphi}$-Bartle integrable $|\mu|$-a.e. $s$. For $x^{*}$ in $\mathcal{R} \mathcal{M}^{*}(\mathbb{R})$, corresponding to $f$ in $L^{\infty}\left(\mathbb{R}, m_{L}\right)$ and for $|\mu|$-a.e. $s$, by (2.15) and Theorem 4.4 ,

$$
\begin{aligned}
\int_{C[0, t]} & \theta(s, x(s)) d x^{*} V_{\varphi}(x) \\
= & x^{*}\left((B a)-\int_{C[0, t]} \theta(s, x(s)) d V_{\varphi}(x)\right) \\
= & x^{*}\left(\int_{\mathbb{R}} \int_{\mathbb{R}} \theta\left(s, u_{1}\right) \frac{1}{\sqrt{(2 \pi)^{2} s(t-s)}} \exp \left\{-\frac{\left(u_{1}-u_{0}\right)^{2}}{2 s}-\frac{\left(u_{2}-u_{1}\right)^{2}}{2(t-s)}\right\}\right. \\
& \left.d \varphi\left(u_{0}\right) d m_{L}\left(u_{1}\right) d m_{L}\left(u_{2}\right)\right) \\
= & \int_{\mathbb{R}} \int_{\mathbb{R}} \int_{\mathbb{R}} f\left(u_{1}\right) \theta\left(s, u_{1}\right) \frac{1}{\sqrt{(2 \pi)^{2} s(t-s)}} \exp \left\{-\frac{\left(u_{1}-u_{0}\right)^{2}}{2 s}-\frac{\left(u_{2}-u_{1}\right)^{2}}{2(t-s)}\right\} \\
& d \varphi\left(u_{0}\right) d m_{L}\left(u_{1}\right) d m_{L}\left(u_{2}\right) \cdot
\end{aligned}
$$

So, we obtain Step (5). By (2.20), we know that Step (7) holds. Hence by [15] Definition 2.1, p. 159], $\theta(s, x(s))$ is $\mu \times V_{\varphi}$-Bartle integrable. 
WIENER MEASURE AND THE MEASURE-VALUED FEYNMAN-KAC FORMULA 4943

Theorem 5.4. (1) $K(m, n, q, j)$ is $\left(\prod_{i=1}^{n} \prod_{j=1}^{j_{i}} \mu\right) \times V_{\varphi}$-Bartle integrable,

(2) for $\prod_{i=1}^{n} \prod_{j=1}^{j_{i}}|\mu|$-a.e. $\left(s_{1,1}, \cdots, s_{n, j_{n}}\right), K(m, n, q, j)\left(\left(s_{1,1}, \cdots, s_{n, j_{n}}\right), \cdot\right)$ is $V_{\varphi}$-Bartle integrable, and

(3) $(B a)-\int_{C[0, t]} K(m, n, q, j)\left(\left(s_{1,1}, \cdots, s_{n, j_{n}}\right), x\right) d V_{\varphi}(x)$ is $\prod_{i=1}^{n} \prod_{j=1}^{j_{i}} \mu$-Bochner integrable.

Proof. By Lemma 5.3, the statement (1) in this theorem holds. From Lemma 5.2, for $\prod_{i=1}^{n} \prod_{j=1}^{j_{i}}|\mu|$-a.e. $\left(s_{1,1}, \cdots, s_{n, j_{n}}\right)$,

$$
\begin{aligned}
& \left|K(m, n, q, j)\left(\left(s_{1,1}, \cdots, s_{n, j_{n}}\right), x\right)\right| \\
& \quad \leq D(m, n, q, j)\left(s_{1,1}, \cdots, s_{n, j_{n}}\right)
\end{aligned}
$$

holds for $\left\|V_{\varphi}\right\|$-a.e. $x$ and $D(m, n, q, j)$ is finite; that is, for $\prod_{i=1}^{n} \prod_{j=1}^{j_{i}}|\mu|$ a.e. $\left(s_{1,1}, \cdots, s_{n, j_{n}}\right), K(m, n, q, j)\left(\left(s_{1,1}, \cdots, s_{n, j_{n}}\right), x\right)$ is $\left\|V_{\varphi}\right\|$-essentially bounded with respect to $x$. Hence, from $(2.14)$, for $\prod_{i=1}^{n} \prod_{j=1}^{j_{i}}|\mu|$-a.e. $\left(s_{1,1}, \cdots, s_{n, j_{n}}\right)$, $K(m, n, q, j)\left(\left(s_{1,1}, \cdots, s_{n, j_{n}}\right), x\right)$ is a $V_{\varphi}$-Bartle integrable function of $x$.

By the classical Fubini theorem, for $x^{*}$ in $\mathcal{R} \mathcal{M}(\mathbb{R})^{*}$,

$$
\begin{array}{r}
x^{*}\left[(B a)-\int_{C[0, t]} K(m, n, q, j)\left(\left(s_{1,1}, \cdots, s_{n, j_{n}}\right), x\right) d V_{\varphi}(x)\right] \\
=\int_{C[0, t]} K(m, n, q, j)\left(\left(s_{1,1}, \cdots, s_{n, j_{n}}\right), x\right) d\left(x^{*} V_{\varphi}\right)(x)
\end{array}
$$

is $\prod_{i=1}^{n} \prod_{j=1}^{j_{i}} \mu$-measurable with respect to $\left(s_{1,1}, \cdots, s_{n, j_{n}}\right)$. That is, $(B a)-$ $\int_{C[0, t]} K(m, n, q, j)\left(\left(s_{1,1}, \cdots, s_{n, j_{n}}\right), x\right) d V_{\varphi}(x)$ is $\prod_{i=1}^{n} \prod_{j=1}^{j_{i}} \mu$-weakly measurable with respect to $\left(s_{1,1}, \cdots, s_{n, j_{n}}\right)$. Since $\mathcal{R} \mathcal{M}(\mathbb{R})$ is separable, by $(2.6)$,

$$
(B a)-\int_{C[0, t]} K(m, n, q, j)\left(\left(s_{1,1}, \cdots, s_{n, j_{n}}\right), x\right) d V_{\varphi}(x)
$$

is $\prod_{i=1}^{n} \prod_{j=1}^{j_{i}} \mu$-measurable with respect to $\left(s_{1,1}, \cdots, s_{n, j_{n}}\right)$. By Lemma 5.2 ,

$$
\begin{gathered}
\int_{\triangle_{q_{n+1} ; j_{1}, \cdots, j_{n}}} \mid(B a)-\int_{C[0, t]} K(m, n, q, j)\left(\left(s_{1,1}, \cdots, s_{n, j_{n}}\right), x\right) \\
d V_{\varphi}(x) \mid(\mathbb{R}) d\left(\prod_{i=1}^{n} \prod_{j=1}^{j_{i}}|\mu|\right)\left(s_{1,1}, \cdots, s_{n, j_{n}}\right) \\
\leq \frac{4}{q_{n+1} !}|\varphi|(\mathbb{R})\left(\prod_{i=0}^{n}\left\|\theta\left(\tau_{i}, \cdot\right)\right\|_{\varphi ; \infty}^{q_{i}}\right)\left(\|\theta\|_{\varphi ; \infty, 1 ; \mu}\right)^{q_{n+1}} \\
<+\infty .
\end{gathered}
$$

Hence, by $(2.8), \int_{C[0, t]} K(m, n, q, j)\left(\left(s_{1,1}, \cdots, s_{n, j_{n}}\right), x\right) d V_{\varphi}(x)$ is $\prod_{i=1}^{n} \prod_{j=1}^{j_{i}} \mu$ Bochner integrable.

At this point, we establish one of our main theorems in this article. The proof of the following theorem is patterned to some extent on earlier work by Johnson and Lapidus in [9], but the present setting requires a number of new concepts and results in the previous parts of this article. 
Theorem 5.5 (A measure-valued Feynman-Kac formula).

$\exp \left\{\int_{[0, t]} \theta(s, x(s)) d \eta(s)\right\}$ is $V_{\varphi}$-Bartle integrable on $C[0, t]$ and for $E$ in $\mathcal{B}(\mathbb{R})$,

$$
\begin{aligned}
& {\left[(B a)-\int_{C[0, t]} \exp \left\{\int_{[0, t]} \theta(s, x(s)) d \eta(s)\right\} d V_{\varphi}(x)\right](E)} \\
& =\sum_{m=0}^{\infty} \sum_{q_{0}+\cdots+q_{n+1}=m} \frac{\prod_{p=0}^{n} c_{p}^{q_{p}}}{\prod_{p=0}^{n} q_{p} !} \sum_{j_{1}+\cdots+j_{n}=q_{n+1}} \int_{\triangle_{q_{n+1} ; j_{1}, \cdots, j_{n}}} \\
& \quad\left[\left(L_{n} \circ L_{n-1} \circ \cdots \circ L_{1}\right)\left(T\left(s_{1,1}, \varphi, \theta(0, \cdot)^{q_{0}}\right)\right)\right](E) d\left(\prod_{i=1}^{n} \prod_{j=1}^{j_{i}} \mu\right)\left(s_{1,1}, \cdots, s_{n, j_{n}}\right) .
\end{aligned}
$$

Moreover,

$$
\begin{aligned}
& \left|(B a)-\int_{C[0, t]} \exp \left\{\int_{[0, t]} \theta(s, x(s)) d \eta(s)\right\} d V_{\varphi}(x)\right|(\mathbb{R}) \\
& \leq 4|\varphi|(\mathbb{R})\left[\exp \left\{\|\theta\|_{\varphi ; \infty, 1 ; \eta}\right\}\right] .
\end{aligned}
$$

Here, for $k=2,3, \cdots, n$,

$$
L_{k}=M_{\theta\left(\tau_{k}\right)^{q_{k}}} \circ S_{\tau_{k}-s_{k, j_{k}}} \circ M_{\theta\left(s_{k, j_{k}}\right)} \circ S_{s_{k, j_{k}}-s_{k, j_{k}-1}} \circ \cdots \circ M_{\theta\left(s_{k, 1}\right)} \circ S_{s_{k, 1}-s_{k, 0}}
$$

and

$$
L_{1}=M_{\theta\left(\tau_{1}\right)^{q_{1}}} \circ S_{\tau_{1}-s_{1, j_{1}}} \circ M_{\theta\left(s_{1, j_{1}}\right)} \circ S_{s_{1, j_{1}}-s_{1, j_{1}-1}} \circ \cdots \circ M_{\theta\left(s_{1,1}\right)} .
$$

Proof. By Theorem 5.1, we have

$$
\begin{aligned}
& \left|\exp \left\{\int_{[0, t]} \theta(s, x(s)) d \eta(s)\right\}\right| \\
& \leq \exp \left\{\int_{[0, t]}\|\theta(s, \cdot)\|_{\varphi ; \infty} d|\eta|(s)\right\} \\
& \quad=\exp \left\{\|\theta\|_{\varphi ; \infty, 1 ; \eta}\right\}
\end{aligned}
$$

for $\left\|V_{\varphi}\right\|$-a.e. $x$.

Hence, by (2.14), Lemma 5.3 and the Fubini theorem, $\exp \left\{\int_{[0, t]} \theta(s, x(s)) d \eta(s)\right\}$ is $V_{\varphi}$-Bartle integrable on $C[0, t]$. Moreover, by $(2.14)$ and $(4.12)$, we have

$$
\begin{aligned}
& \left|(B a)-\int_{C[0, t]} \exp \left\{\int_{[0, t]} \theta(s, x(s)) d \eta(s)\right\} d V_{\varphi}(x)\right|(\mathbb{R}) \\
& \leq 4|\varphi|(\mathbb{R}) \exp \left\{\|\theta\|_{\varphi ; \infty, 1 ; \eta}\right\} ;
\end{aligned}
$$

so, we obtain the inequality (5.17). Since for any nonnegative integer $n$,

$$
\left|\left(\int_{[0, t]} \theta(s, x(s)) d \eta(s)\right)^{n}\right| \leq\left(\|\theta\|_{\varphi ; \infty, 1 ; \eta}\right)^{n}
$$

for $\left\|V_{\varphi}\right\|$-a.e. $x$, by $(2.17),\left(\int_{[0, t]} \theta(s, x(s)) d \eta(s)\right)^{n}$ is $V_{\varphi}$-Bartle integrable. 
Now, we prove the equality (5.16). For $E$ in $\mathcal{B}(\mathbb{R})$,

$$
\begin{aligned}
& {\left[(B a)-\int_{C[0, t]} \exp \left\{\int_{[0, t]} \theta(s, x(s)) d \eta(s)\right\} d V_{\varphi}(x)\right](E)} \\
& \stackrel{(1)}{=}\left[(B a)-\int_{C[0, t]} \sum_{m=0}^{\infty} \frac{1}{m !}\left(\int_{[0, t]} \theta(s, x(s)) d \eta(s)\right)^{m} d V_{\varphi}(x)\right](E) \\
& \stackrel{(2)}{=} \sum_{m=0}^{\infty} \frac{1}{m !}\left[(B a)-\int_{C[0, t]}\left(\int_{[0, t]} \theta(s, x(s)) d \eta(s)\right)^{m} d V_{\varphi}(x)\right](E) \\
& \stackrel{(3)}{=} \sum_{m=0}^{\infty} \frac{1}{m !}\left[(B a)-\int_{C[0, t]}\left(\sum_{p=0}^{n} c_{p} \theta\left(\tau_{p}, x\left(\tau_{p}\right)\right)\right.\right. \\
& \left.\left.+\int_{[0, t]} \theta(s, x(s)) d \mu(s)\right)^{m} d V_{\varphi}(x)\right](E) \\
& \stackrel{(4)}{=} \sum_{m=0}^{\infty} \sum_{q_{0}+\cdots+q_{n+1}=m} \frac{\prod_{j=0}^{n} c_{j}^{q_{j}}}{\prod_{j=0}^{n+1} q_{j} !}\left[(B a)-\int_{C[0, t]}\left(\prod_{j=0}^{n} \theta\left(\tau_{j}, x\left(\tau_{j}\right)\right)^{q_{j}}\right)\right. \\
& \left.\left(\int_{[0, t]} \theta(s, x(s)) d \mu(s)\right)^{q_{n+1}} d V_{\varphi}(x)\right](E) \\
& \stackrel{(5)}{=} \sum_{m=0}^{\infty} \sum_{q_{0}+\cdots+q_{n+1}=m} \frac{\prod_{j=0}^{n} c_{j}^{q_{j}}}{\prod_{j=0}^{n+1} q_{j} !}\left[(B a)-\int_{C[0, t]}\left(\prod_{j=0}^{n} \theta\left(\tau_{j}, x\left(\tau_{j}\right)\right)^{q_{j}}\right)\left(q_{n+1}\right)\right. \text { ! } \\
& \left.\left(\int_{\triangle_{q_{n+1}}} \prod_{j=1}^{q_{n+1}} \theta\left(s_{j}, x\left(s_{j}\right)\right) d\left(\prod_{j=1}^{q_{n+1}} \mu\right)\left(s_{1}, s_{2}, \cdots, s_{q_{n+1}}\right)\right) d V_{\varphi}(x)\right](E) \\
& \stackrel{(6)}{=} \sum_{m=0}^{\infty} \sum_{q_{0}+\cdots+q_{n+1}=m} \frac{\prod_{j=0}^{n} c_{j}^{q_{j}}}{\prod_{j=0}^{n} q_{j} !} \sum_{j_{1}+\cdots+j_{n}=q_{n+1}} \\
& {\left[(B a)-\int_{C[0, t]} \int_{\triangle_{q_{n+1} ; j_{1}, \cdots, j_{n}}}\right.} \\
& \theta\left(\tau_{0}, x\left(\tau_{0}\right)\right)^{q_{0}}\left\{\prod_{i=1}^{n}\left(\prod_{j=1}^{j_{i}} \theta\left(s_{i, j}, x\left(s_{i, j}\right)\right)\right) \theta\left(s_{i, j_{i}+1}, x\left(s_{i, j_{i}+1}\right)\right)^{q_{i}}\right\} \\
& \left.d\left(\prod_{i=1}^{n} \prod_{j=1}^{j_{i}} \mu\right)\left(s_{1,1}, \cdots, s_{n, j_{n}}\right) d V_{\varphi}(x)\right](E) \\
& \stackrel{(7)}{=} \sum_{m=0}^{\infty} \sum_{q_{0}+\cdots+q_{n+1}=m} \frac{\prod_{j=0}^{n} c_{j}^{q_{j}}}{\prod_{j=0}^{n} q_{j} !} \sum_{j_{1}+\cdots+j_{n}=q_{n+1}} \int_{\triangle_{q_{n+1} ; j_{1}, \cdots, j_{n}}}[(B a) \\
& -\int_{C[0, t]} \theta\left(\tau_{0}, x\left(\tau_{0}\right)\right)^{q_{0}}\left\{\prod_{i=1}^{n}\left(\prod_{j=1}^{j_{i}} \theta\left(s_{i, j}, x\left(s_{i, j}\right)\right)\right) \theta\left(s_{i, j_{i}+1}, x\left(s_{i, j_{i}+1}\right)\right)^{q_{i}}\right\} \\
& \left.d V_{\varphi}(x)\right](E) d\left(\prod_{i=1}^{n} \prod_{j=1}^{j_{i}} \mu\right)\left(s_{1,1}, \cdots, s_{n, j_{n}}\right)
\end{aligned}
$$




$$
\begin{aligned}
& \stackrel{(8)}{=} \sum_{m=0}^{\infty} \sum_{q_{0}+\cdots+q_{n+1}=m} \frac{\prod_{j=0}^{n} c_{j}^{q_{j}}}{\prod_{j=0}^{n} q_{j} !} \sum_{j_{1}+\cdots+j_{n}=q_{n+1}} \int_{\triangle_{q_{n+1} ; j_{1}, \cdots, j_{n}}}(2 \pi)^{-\frac{1}{2}\left(q_{n+1}+n\right)} \\
& \left(\prod_{i=1}^{n} \prod_{j=1}^{j_{i}+1}\left(s_{i, j}-s_{i, j-1}\right)\right)^{-\frac{1}{2}} \int_{\mathbb{R}} \int_{\mathbb{R}^{q_{n+1}+n}} \theta\left(0, u_{0,0}\right)^{q_{0}}\left(\prod_{i=1}^{n}\left(\prod_{j=1}^{j_{i}} \theta\left(s_{i, j}, u_{i, j}\right)\right)\right. \\
& \left.\theta\left(s_{i, j_{i}+1}, u_{i, j_{i}+1}\right)^{q_{i}}\right) \chi_{E}\left(u_{n, j_{n}+1}\right) \exp \left\{-\frac{1}{2} \sum_{i=1}^{n} \sum_{j=1}^{j_{i}+1} \frac{\left(u_{i, j}-u_{i, j-1}\right)^{2}}{s_{i, j}-s_{i, j-1}}\right\} \\
& d\left(\prod_{i=1}^{n} \prod_{j=1}^{j_{i}+1} m_{L}\right)\left(u_{1,1}, \cdots, u_{n, j_{n}+1}\right) d \varphi\left(u_{0,0}\right) d\left(\prod_{i=1}^{n} \prod_{j=1}^{j_{i}} \mu\right)\left(s_{1,1}, \cdots, s_{n, j_{n}+1}\right) \\
& \stackrel{(9)}{=} \sum_{m=0}^{\infty} \sum_{q_{0}+\cdots+q_{n+1}=m} \frac{\prod_{j=1}^{n} c_{j}^{q_{j}}}{\prod_{j=1}^{n} q_{j} !} \sum_{j_{1}+\cdots+j_{n}=q_{n+1}} \int_{\triangle_{q_{n+1} ; j_{1}, \cdots, j_{n}}}\left[\left(L_{n} \circ L_{n-1} \circ \cdots \circ L_{1}\right)\right. \\
& \left.\left(T\left(s_{1,1} ; \varphi ; \theta(0, \cdot)^{q_{0}}\right)\right)\right](E) d\left(\prod_{i=1}^{n} \prod_{j=1}^{j_{i}} \mu\right)\left(s_{1,1}, \cdots, s_{n, j_{n}}\right) .
\end{aligned}
$$

By the Taylor expansion, we have Step (1). Letting

$$
f_{n}(x)=\sum_{m=0}^{n} \frac{1}{m !}\left(\int_{[0, t]} \theta(s, x(s)) d \eta(s)\right)^{m}
$$

on $C[0, t]$ for any nonnegative integer $n,\left\langle f_{n}\right\rangle$ converges to

$$
\exp \left\{\int_{[0, t]} \theta(s, x(s)) d \eta(s)\right\}
$$

for $\left\|V_{\varphi}\right\|$-a.e. $x$ and $\exp \left\{\int_{[0, t]} \theta(s, x(s)) d \eta(s)\right\}$ is $V_{\varphi}$-Bartle integrable, by (2.17), we obtain Step (2). Step (3) follows from the elementary computation of an integral. From the multinomial expansion,

$$
\left(a_{0}+a_{1}+\cdots+a_{n}\right)^{m}=\sum_{q_{0}+\cdots+q_{n}=m} \frac{m !}{q_{0} ! \cdots q_{n} !} a_{0}^{q_{0}} \cdots a_{n}^{q_{n}},
$$

we have Step (4). Let $\triangle_{q_{n+1}}=\left\{\left(s_{1}, \cdots, s_{q_{n+1}}\right)\right.$ in $[0, t]^{q_{n+1}}$

$\left.\mid 0<s_{1}<s_{2}<\cdots<s_{q_{n+1}}<t\right\}$, let $\mathcal{P}$ be the set of all permutations on $\left\{1,2, \cdots, q_{n+1}\right\}$, for $\sigma$ in $\mathcal{P}$, let $\triangle_{q_{n+1} ; \sigma}=\left\{\left(s_{\sigma(1)}, \cdots, s_{\sigma\left(q_{n+1}\right)}\right)\right.$ in $[0, t]^{q_{n+1}}$ | $\left(s_{1}, \cdots, s_{q_{n+1}}\right)$ is in $\left.\triangle_{q_{n+1}}\right\}$, and let $N=\left\{\left(s_{1}, \cdots, s_{q_{n+1}}\right)\right.$ in $[0, t]^{q_{n+1}} \mid$ either for some $1 \leq i, j \leq q_{n+1}$ with $i \neq j, s_{i}=s_{j}$ or for some $1 \leq i \leq q_{n+1}, s_{i}=0$ or $\left.s_{i}=t\right\}$. Then for $\sigma_{1} \neq \sigma_{2}$ in $\mathcal{P}, \triangle_{q_{n+1} ; \sigma_{1}} \cap \triangle_{q_{n+1} ; \sigma_{2}}=\emptyset$, for $\sigma$ in $\mathcal{P}, \triangle_{q_{n+1} ; \sigma}$ is Borel measurable and $[0, t]^{q_{n+1}}=N \cup\left(\bigcup_{\sigma \in \mathcal{P}} \triangle_{q_{n+1} ; \sigma}\right)$. Since $\mu$ has no atoms, by the Fubini theorem, $\left(\prod_{i=1}^{q_{n+1}} \mu\right)(N)=0$ and for $\sigma$ in $\mathcal{P}$,

$$
\begin{aligned}
\int_{\triangle_{q_{n+1}} ; \sigma} & \prod_{i=1}^{q_{n+1}} \theta\left(s_{i}, x\left(s_{i}\right)\right) d\left(\prod_{i=1}^{q_{n+1}} \mu\right)\left(s_{1}, \cdots, s_{q_{n+1}}\right) \\
= & \int_{\triangle_{q_{n+1}}} \prod_{i=1}^{q_{n+1}} \theta\left(s_{i}, x\left(s_{i}\right)\right) d\left(\prod_{i=1}^{q_{n+1}} \mu\right)\left(s_{1}, \cdots, s_{q_{n+1}}\right) .
\end{aligned}
$$


Hence, Step (5) is true. Let $N^{*}=\left\{\left(s_{1}, s_{2}, \cdots, s_{q_{n+1}}\right) \mid 0<s_{1}<s_{2}<\cdots<\right.$ $s_{j_{1}} \leq \tau_{1} \leq s_{j_{1}+1}<s_{j_{1}+2}<\cdots<s_{j_{1}+j_{2}} \leq \tau_{2} \leq s_{j_{1}+j_{2}+1}<\cdots<s_{j_{1}+\cdots+j_{n}} \leq$ $\tau_{n}=t$ and for some $k=1,2, \cdots, n$ and for some $\left.j=1,2, \cdots, q_{n+1}, \tau_{k}=s_{j}\right\}$. Then for $\left(j_{1}, \cdots, j_{n}\right) \neq\left(j_{1}^{\prime}, \cdots, j_{n}^{\prime}\right), \triangle_{q_{n+1} ; j_{1}, \cdots, j_{n}} \cap \triangle_{q_{n+1} ; j_{1}^{\prime}, \cdots, j_{n}^{\prime}}=\emptyset$, for all $j_{1}, j_{2}, \cdots, j_{n}$ with $j_{1}+\cdots+j_{n}=q_{n+1}, \triangle_{q_{n+1} ; j_{1}, \cdots, j_{n}}$ and $N^{*}$ are Borel measurable, $\left(\prod_{i=1}^{q_{n+1}} \mu\right)\left(N^{*}\right)=0$ and $\triangle_{q_{n+1}}=N^{*} \cup\left(\bigcup_{j_{1}+\cdots+j_{n}=q_{n+1}}\right.$ $\left.\triangle_{q_{n+1} ; j_{1}, \cdots, j_{n}}\right)$. Therefore, by the relabelling $s_{j_{1}+\cdots+j_{k-1}+l}=s_{k-1, l}$ for $k=0,1, \cdots$, $n$ and $l=0,1, \cdots, j_{k}$, we deduce that Step (6) holds. By Theorem 2.1, Theorem 2.3 and Theorem 5.4, we obtain Step (7). For $i=1,2, \cdots, n$, let $u_{i, j_{i}+1}=u_{i+1,0}$. By the relabelling as follows: for $k=0,1, \cdots, n, \tau_{k}=s_{k, j_{k}+1}=s_{k+1,0}$ and by Theorem 4.4, Step (8) holds. By the definitions of $S, M$ and $T$ in $(\mathrm{H})$ of section 2, we have Step (9). Thus, the proof of this theorem is finished.

From Theorem 5.5, directly we deduce the following corollaries.

Corollary 5.6. In Theorem 5.5, we assume that $\eta=\mu$, an arbitrary continuous measure on $[0, t]$. Then for $E$ in $\mathcal{B}(\mathbb{R})$,

$$
\begin{aligned}
{[(B a)-} & \left.\int_{C[0, t]} \exp \left\{\int_{[0, t]} \theta(s, x(s)) d \eta(s)\right\} d V_{\varphi}(x)\right](E) \\
= & \sum_{m=0}^{\infty} \int_{\triangle_{m}}\left[\left(S_{t-s_{m}} \circ M_{\theta\left(s_{m}\right)} \circ \cdots \circ S_{s_{2}-s_{1}} \circ M_{\theta\left(s_{1}\right)}\right)\left(T\left(s_{1}, \varphi, \theta^{0} \equiv 1\right)\right)\right] \\
& (E) d\left(\prod_{i=1}^{m} \mu\right)\left(s_{1}, s_{2}, \cdots, s_{m}\right)
\end{aligned}
$$

where $\triangle_{m}=\left\{\left(s_{1}, s_{2}, \cdots, s_{m}\right)\right.$ in $\left.[0, t]^{m} \mid 0<s_{1}<s_{2}<\cdots<s_{m}<t\right\}$.

Corollary 5.7. In Theorem 5.5, we assume that $\eta=\nu=\sum_{p=0}^{n} c_{p} \delta_{\tau_{p}}$, a discrete measure on $[0, t]$ with finite support. Then for $E$ in $\mathcal{B}(\mathbb{R})$,

$$
\begin{aligned}
{[(B a)-} & \left.\int_{C[0, t]} \exp \left\{\int_{[0, t]} \theta(s, x(s)) d \eta(s)\right\} d V_{\varphi}(x)\right](E) \\
= & \sum_{m=0}^{\infty} \sum_{q_{0}+\cdots+q_{n}=m} \frac{\prod_{p=0}^{n} c_{p}^{q_{p}}}{\prod_{p=0}^{n} q_{p} !}\left[\left(M_{\theta\left(\tau_{n}\right)^{q_{n}}} \circ S_{\tau_{n}-\tau_{n-1}} \circ \cdots \circ S_{\tau_{2}-\tau_{1}} \circ M_{\theta\left(\tau_{1}\right)^{q_{1}}}\right)\right. \\
& \left.\left(T\left(\tau_{1}, \varphi, \theta(0, \cdot)^{q_{0}}\right)\right)\right](E) .
\end{aligned}
$$


Corollary 5.8. In Theorem 5.5, we assume that $c_{n}=0$. Then for $E$ in $\mathcal{B}(\mathbb{R})$,

$$
\begin{aligned}
& {\left[(B a)-\int_{C[0, t]} \exp \left\{\int_{[0, t]} \theta(s, x(s)) d \eta(s)\right\} d V_{\varphi}(x)\right](E)} \\
& =\sum_{m=0}^{\infty} \sum_{q_{0}+\cdots+q_{n}=m} \frac{\prod_{p=0}^{n-1} c_{p}^{q_{p}}}{\prod_{p=0}^{n-1} q_{p} !} \sum_{j_{1}+\cdots+j_{n}=q_{n}} \int_{\triangle_{q_{n} ; j_{1}, \cdots, j_{n}}} \\
& {\left[\left(\left(S_{t-s_{n, j_{n}}} \circ M_{\theta\left(s_{n, j_{n}}\right)} \circ \cdots \circ S_{s_{n, 1}-\tau_{n-1}}\right) \circ L_{n-1} \circ \cdots \circ L_{1}\right)\right.} \\
& \left.\left(T\left(s_{1,1}, \varphi, \theta(0, \cdot)^{q_{0}}\right)\right)\right](E) d\left(\prod_{i=1}^{n} \prod_{j=1}^{j_{i}} \mu\right)\left(s_{1,1}, \cdots, s_{n, j_{n}}\right) .
\end{aligned}
$$

\section{A Volterra integral equation for the MEASURE-VALUED FEYNMAN-KaC FORMUla}

In this section, we prove that the equality (5.16) in Theorem 5.5, satisfies a suitable Volterra integral equation (see [10], [12, 13], 14]).

Throughout this section, let $0=\tau_{0}<\tau_{1}<\cdots<\tau_{n}=t<\tilde{t}$ and let $\eta$ be a Borel measure on $[0, \widetilde{t}]$ such that $\eta=\mu+\nu$ where $\mu$ is the continuous part of $\eta$ and $\nu=\sum_{p=0}^{n} c_{p} \delta_{\tau_{p}}$; furthermore, let $\theta$ be in $L_{\varphi ; \infty, 1 ; \eta}^{\widetilde{t}}$. Let

$$
u\left(t^{\prime}\right)=(B a)-\int_{C\left[0, t^{\prime}\right]} \exp \left\{\int_{\left[0, t^{\prime}\right]} \theta(s, x(s)) d \eta(s)\right\} d V_{\varphi}(x)
$$

for $t<t^{\prime} \leq \widetilde{t}$.

The following theorem is the counterpart for the measure-valued measure $V_{\varphi}$ of the integral equation for the Feynman-Kac formula with Lebesgue-Stieltjes measure, obtained by Lapidus in [12, [13, 14] and for the Feynman-Kac formula with an operator-valued measure, obtained by Kluvanek in [10].

Theorem 6.1 (The measure-valued Feynman-Kac formula). For $t<t^{\prime} \leq \widetilde{t}, u\left(t^{\prime}\right)$ satisfies a Volterra integral equation, that is,

$$
u\left(t^{\prime}\right)=S_{t^{\prime}-t}(u(t))+(B o)-\int_{\left(t, t^{\prime}\right]}\left(S_{t^{\prime}-s} \circ M_{\theta(s)}\right) u(s) d \mu(s) .
$$

Proof. By Corollary 5.8, for $t<s \leq \tilde{t}$,

$$
\begin{aligned}
u(s)= & \left.\sum_{m=0}^{\infty} \sum_{q_{0}+\cdots+q_{n+1}=m} \frac{\prod_{p=0}^{n} c_{p}^{q_{p}}}{\prod_{p=0}^{n} q_{p} !} \sum_{j_{1}+\cdots+j_{n+1}=q_{n+1}}(B o)-\int_{\triangle_{q_{n+1} ; j_{1}, \cdots, j_{n+1}}^{(s)}} \circ M_{\theta\left(s_{n+1, j_{n+1}}\right)} \circ \cdots \circ S_{s_{n+1,1}-s_{n+1,0}} \circ L_{n} \circ \cdots \circ L_{1}\right] \\
& {\left[S_{s-s_{n+1, j_{n+1}}} \circ{ }^{n+1} j_{i}\right.} \\
& \left(T\left(s_{1,1}, \varphi, \theta(0, \cdot)^{q_{0}}\right)\right) d\left(\prod_{i=1}^{n+1} \prod_{j=1}^{\mu} \mu\left(s_{1,1}, \cdots, s_{n+1, j_{n+1}}\right),\right.
\end{aligned}
$$


WIENER MEASURE AND THE MEASURE-VALUED FEYNMAN-KAC FORMULA 4949

where $\triangle_{q_{n+1} ; j_{1}, \cdots, j_{n+1}}^{(s)}=\left\{\left(s_{1,1}, \cdots, s_{n+1, j_{n+1}}\right) \mid 0=s_{0,0}=\tau_{0}<s_{1,1}<\cdots<s_{1, j_{1}}<\right.$ $\left.\tau_{1}<s_{2,1}<\cdots<\tau_{n}<s_{n+1,1}<\cdots<s_{n+1, j_{n+1}}<s\right\}$ and for $k=1,2, \cdots, n, L_{k}$ is given in Theorem 5.5. For $t<s \leq \widetilde{t}$, let

$$
\begin{aligned}
& Y\left(s ; q_{0}, \cdots, q_{n+1} ; j_{1}, \cdots, j_{n+1}\right) \\
& =(B o)-\int_{\triangle_{q_{n+1} ; j_{1}, \cdots, j_{n+1}}^{(s)}}\left[S_{s-s_{n+1, j_{n+1}}} \circ M_{\theta\left(s_{n+1, j_{n+1}}\right)} \circ \cdots \circ S_{s_{n+1,1}-s_{n+1,0}}\right. \\
& \left.\quad \circ L_{n} \circ \cdots \circ L_{1}\right]\left(T\left(s_{1,1}, \varphi, \theta(0, \cdot)^{q_{0}}\right)\right) d\left(\prod_{i=1}^{n+1} \prod_{j=1}^{j_{i}} \mu\right)\left(s_{1,1}, \cdots, s_{n+1, j_{n+1}}\right) .
\end{aligned}
$$

For $t<t^{\prime} \leq \widetilde{t}$, let

$$
\begin{aligned}
& u_{1}\left(t^{\prime}\right)=\sum_{m=1}^{\infty} \sum_{q_{0}+\cdots+q_{n}=m} \frac{\prod_{p=0}^{n} c_{p}^{q_{p}}}{\prod_{p=0}^{n} q_{p} !} Y\left(t^{\prime} ; q_{0}, \cdots, q_{n}, 0 ; 0,0, \cdots, 0\right), \\
& u_{2}\left(t^{\prime}\right)=\sum_{m=1}^{\infty} \sum_{\substack{q_{0}+\cdots+q_{n+1}=m \\
q_{n+1} \geq 1}} \frac{\prod_{p=0}^{n} c_{p}^{q_{p}}}{\prod_{p=0}^{n} q_{p} !} \sum_{j_{1}+\cdots+j_{n}=q_{n+1}} \\
& Y\left(t^{\prime} ; q_{0}, \cdots, q_{n}, q_{n+1} ; j_{1}, \cdots, j_{n}, 0\right) \\
& u_{3}\left(t^{\prime}\right)=\sum_{m=1}^{\infty} \sum_{\substack{q_{0}+\cdots+q_{n+1}=m \\
q_{n+1} \geq 1}} \frac{\prod_{p=0}^{n} c_{p}^{q_{p}}}{\prod_{p=0}^{n} q_{p} !} \sum_{\substack{j_{1}+\cdots+j_{n+1}=q_{n+1} \\
j_{n+1} \geq 1}} \\
& Y\left(t^{\prime} ; q_{0}, \cdots, q_{n+1} ; j_{1}, \cdots, j_{n+1}\right) \text {. }
\end{aligned}
$$

Since $Y\left(t^{\prime} ; q_{0}, \cdots, q_{n}, q_{n+1} ; j_{1}, \cdots, j_{n}, 0\right)=S_{t^{\prime}-t}\left(Y\left(t^{\prime} ; q_{0}, \cdots, q_{n}, q_{n+1} ; j_{1}, \cdots, j_{n}\right)\right)$, by (2.32),

$$
\begin{aligned}
S_{t^{\prime}}(\varphi)+ & u_{1}\left(t^{\prime}\right)+u_{2}\left(t^{\prime}\right) \\
= & S_{t^{\prime}-t}\left(S_{t}(\varphi)+\sum_{m=1}^{\infty} \sum_{q_{0}+\cdots+q_{n+1}=m} \frac{\prod_{p=0}^{n} c_{p}^{q_{p}}}{\prod_{p=0}^{n} q_{p} !} \sum_{j_{1}+\cdots+j_{n}=q_{n+1}}\right. \\
& \left.Y\left(t^{\prime} ; q_{0}, \cdots, q_{n}, q_{n+1} ; j_{1}, \cdots, j_{n}\right)\right) \\
= & S_{t^{\prime}-t}(u(t)) .
\end{aligned}
$$


On the other hand,

$$
\begin{aligned}
(B o)- & \int_{\left(t, t^{\prime}\right]}\left(S_{t^{\prime}-s} \circ M_{\theta(s)}\right)(u(s)) d \mu(s) \\
& \stackrel{(1)}{=} \sum_{m=0}^{\infty} \sum_{q_{0}+\cdots+q_{n+1}=m} \frac{\prod_{p=0}^{n} c_{p}^{q_{p}}}{\prod_{p=0}^{n} q_{p} !} \sum_{j_{1}+\cdots+j_{n+1}=q_{n+1}}(B o) \\
& -\int_{\left(t, t^{\prime}\right]}\left(S_{t^{\prime}-s} \circ M_{\theta(s)}\right)\left(Y\left(s ; q_{0}, \cdots, q_{n+1} ; j_{1}, \cdots, j_{n+1}\right)\right) d \mu(s) \\
& \stackrel{(2)}{=} \sum_{m=0}^{\infty} \sum_{q_{0}+\cdots+q_{n+1}=m} \frac{\prod_{p=0}^{n} c_{p}^{q_{p}}}{\prod_{p=0}^{n} q_{p} !} \sum_{j_{1}+\cdots+j_{n+1}=q_{n+1}} \\
& Y\left(t^{\prime} ; q_{0}, \cdots, q_{n+1}+1 ; j_{1}, \cdots, j_{n+1}+1\right) \\
& \stackrel{(3)}{=} \sum_{m^{\prime}=1}^{\infty} \sum_{q_{0}^{\prime}+q_{1}^{\prime}+\cdots+q_{n+1}^{\prime}=m^{\prime}} \frac{\prod_{p=0}^{n} c_{p=0}^{q_{p}^{\prime}}}{\prod_{p}^{n} !} \sum_{j_{1}^{\prime}+\cdots+j_{n+1}^{\prime}=q_{n+1}^{\prime}} \\
& Y\left(t^{\prime} ; q_{0}^{\prime}, \cdots, q_{n+1}^{\prime} ; j_{1}^{\prime}, \cdots, j_{n+1}^{\prime}\right) \\
= & u_{3}\left(t^{\prime}\right) .
\end{aligned}
$$

Step (1) follows from (2.17). By an elementary calculation, we have Step (2). If $q_{n+1} \geq 1$, then the condition " $m=0$ " has no meaning; so by making the substitution $j_{k}=j_{k}^{\prime}(1 \leq k \leq n), j_{n+1}+1=j_{n+1}^{\prime}, q_{k}=q_{k}^{\prime}(1 \leq k \leq n)$, $q_{n+1}+1=q_{n+1}^{\prime}$ and $m+1=m^{\prime}$, we obtain Step (3).

Hence, for $t<t^{\prime} \leq \widetilde{t}$,

$$
\begin{aligned}
u\left(t^{\prime}\right) & =\left(S_{t^{\prime}}(\varphi)+u_{1}\left(t^{\prime}\right)+u_{2}\left(t^{\prime}\right)\right)+u_{3}\left(t^{\prime}\right) \\
& =S_{t^{\prime}-t}(u(t))+(B o)-\int_{\left(t, t^{\prime}\right]}\left(S_{t^{\prime}-s} \circ M_{\theta(s)}\right)(u(s)) d \mu(s),
\end{aligned}
$$

as desired.

Corollary 6.2. Under the assumptions in Corollary 5.6, for $0<t^{\prime} \leq \widetilde{t}, u\left(t^{\prime}\right)$ satisfies a Volterra integral equation, that is,

$$
u\left(t^{\prime}\right)=S_{t^{\prime}}(\varphi)+(B o)-\int_{\left(0, t^{\prime}\right]}\left(S_{t^{\prime}-s} \circ M_{\theta(s)}\right)(u(s)) d \mu(s) .
$$

Corollary 6.3. Under the assumptions in Corollary 5.7, for $0<t^{\prime} \leq \widetilde{t}$,

$$
\begin{gathered}
u\left(t^{\prime}\right)=\sum_{m=0}^{\infty} \sum_{q_{0}+\cdots+q_{n}=m} \frac{\prod_{p=0}^{n} c_{p}^{q_{p}}}{\prod_{p=0}^{n} q_{p} !}\left[S_{t^{\prime}-t} \circ M_{\theta\left(\tau_{n}\right)^{q_{n}}} \circ S_{\tau_{n}-\tau_{n-1}} \circ \cdots\right. \\
\left.\circ S_{\tau_{2}-\tau_{1}} \circ M_{\theta\left(\tau_{1}\right)^{q_{1}}}\right]\left(T\left(\tau_{1}, \varphi, \theta(0, \cdot)^{q_{0}}\right)\right), \\
u\left(t^{\prime}\right)=S_{t^{\prime}-t}(u(t))
\end{gathered}
$$

and

$$
\begin{aligned}
(B o) & -\int_{\left(t, t^{\prime}\right]}\left(S_{t^{\prime}-s} \circ M_{\theta(s)}\right)(u(s)) d \mu(s) \\
& =0, \text { a zero operator. }
\end{aligned}
$$




\section{ACKNOWLEDGEMENT}

The authors would like to thank professor Lapidus of the University of California in Riverside for valuable suggestions and comments which led to a number of improvements.

\section{REFERENCES}

[1] Burrill, C. W., Measure, integration and probability, McGraw-Hill, New York, 1972. MR 56:15862

[2] Cameron, R. H. and Storvick, D. A., An operator-valued function space integral and a related integral equation, J. Math. Mech. 18, 1968, 517-552. MR 38:4643

[3] Cohn, D. L., Measure theory, Birkhäuser, Boston, 1980. MR 81k:28001

[4] Diestel, J. and Uhl, J. J., Vector measures, Mathematical Survey, Amer. Math. Soc., 1977. MR 56:12216

[5] Dunford, N. and Schwartz, J. T., Linear Operators, part I, General Theory, Pure and Applied Mathematics, Vol. VII, Wiley Interscience, New York, 1958. MR 22:8302

[6] Halmos, P. R., Measure Theory, Springer-Verlag, New York, 1950. MR 11:504d

[7] Hewitt, E. and Stromberg, K., Real and Abstract Analysis, Springer-Verlag, New York, 1965. MR 32:5826

[8] Johnson, G. W. and Lapidus, M. L., Generalized Dyson series, generalized Feynman diagrams, the Feynman integral and Feynman's operational calculus, Memoirs Amer. Math. Soc., 62, No. 351, 1986, 1-78. MR 88f:81034

[9] Johnson, G. W. and Lapidus, M. L., The Feynman integral and Feynman's operational calculus, Oxford Mathematical Monographs, Oxford Univ. Press, 2000. MR 2001i:58015

[10] Kluvanek, I., Operator valued measures and perturbations of semi-groups, Arch. Rational Mech. Anal. 81-82, 1983, 161-180. MR 84j:28019

[11] Kluvanek, I. and Knowles, G., Vector measures and control systems, Math. Studies, No 20, Amsterdam, North-Holland, 1975. MR 58:17033

[12] Lapidus, M. L., The Feynman-Kac formula with a Lebesgue-Stieltjes measure and Feynman's operational calculus, Stud. Appl. Math., 76, 1987, 93-132. MR 89j:81057

[13] Lapidus, M. L., Strong product integration of measures and the Feynman-Kac formula with a Lebesgue-Stieltjes measure, Circ. Math. Palermo (2) Suppl. 17 (1987), 271-312. MR 90c:28021

[14] Lapidus, M. L., The Feynman-Kac formula with a Lebesgue-Stieltjes measure: An integral equation in the general case, Integral Equations and Operator Theory, 12 (1989), 163-210. MR 92e:47064

[15] Lewis, D. R., Integration with respect to vector measure, Pacific J. Math., 33, No 1, 1970, 157-165. MR 41:3706

[16] Novinger, W. P., Mean convergence in $L^{p}$ space, Proc. Amer. Math. Soc., 34, 1972, 627628. MR 45:3665

[17] Okikiolu, G. G., Aspect of the theory of bounded linear operators in $L_{p}$ space, Academic Press, London, 1971. MR 56:3581

[18] Parthasarathy, K. R., Probability measures on metric spaces, Academic Press, New York, 1967. MR 37:2271

[19] Rudin, W., Real and complex analysis, 3rd ed., McGraw-Hill, New York, 1987. MR 88k:00002

[20] Wiener, N., Differential space, J. Math. Phys., 2, 1923, 131-174.

[21] Yeh, J., Inversion of conditional expectations, Pacific J. Math., 52, no. 2, 1974, 631-640. MR 51:1896

[22] Yeh, J., Stochastic processes and the Wiener integral, Marcel Deckker, New York, 1973. MR 57:14166

[23] Yosida, K., Functional Analysis, 4th Edition, Springer-Verlag Berlin, 1974. MR 50:2851

Department of Mathematics, Han Nam University, Taejon 306-791, Korea

E-mail address: ksr@math.hannam.ac.kr

Department of Mathematics, Han Nam University, Taejon 306-791, Korea

E-mail address: mki@mail.hannam.ac.kr 